\title{
The Visible and Infrared Survey Telescope for Astronomy (VISTA): Design, technical overview, and performance
}

\author{
Will Sutherland ${ }^{1}$, Jim Emerson ${ }^{1}$, Gavin Dalton ${ }^{2,3}$, Eli Atad-Ettedgui ${ }^{4,5}$, Steven Beard ${ }^{4}$, Richard Bennett ${ }^{4}$, \\ Naidu Bezawada ${ }^{4}$, Andrew Born ${ }^{4}$, Martin Caldwell ${ }^{2}$, Paul Clark ${ }^{6}$, Simon Craig ${ }^{7}$, David Henry ${ }^{4}$, Paul Jeffers ${ }^{7}$, \\ Bryan Little ${ }^{4}$, Alistair McPherson ${ }^{8}$, John Murray ${ }^{4}$, Malcolm Stewart ${ }^{9}$, Brian Stobie ${ }^{4}$, David Terrett ${ }^{2}$, Kim Ward $^{2}$, \\ Martin Whalley ${ }^{2}$, and Guy Woodhouse ${ }^{2}$ \\ ${ }^{1}$ School of Physics and Astronomy, Queen Mary University of London, Mile End Rd, London E1 4NS, UK \\ e-mail: w. j. sutherland@qmul.ac.uk \\ 2 RAL Space, Harwell Oxford, Didcot, Oxfordshire OX11 0QX, UK \\ 3 Astrophysics, University of Oxford, Keble Road, Oxford OX1 3RH, UK \\ ${ }^{4}$ UK Astronomy Technology Centre, Royal Observatory, Blackford Hill, Edinburgh EH9 3HJ, UK \\ 5 Senior Optical Consultant, 9 Abercorn Road, Edinburgh EH8 7DD, UK \\ 6 Centre for Astronomical Instrumentation, University of Durham, South Road, Durham DH1 3LE, UK \\ 7 National Solar Observatory, NSO/DKIST, 950 N. Cherry Avenue, Tucson, AZ 85719, USA \\ 8 SKA Organisation, Jodrell Bank Observatory, Lower Withington, Macclesfield, Cheshire SK11 9DL, UK \\ 9 Solaire Systems, 55/10 Bath Street, Edinburgh EH15 1HE, UK
}

Received 12 September 2014 / Accepted 15 December 2014

\section{ABSTRACT}

\begin{abstract}
The Visible and Infrared Survey Telescope for Astronomy (VISTA) is the 4-m wide-field survey telescope at ESO's Paranal Observatory, equipped with the world's largest near-infrared imaging camera (VISTA IR Camera, VIRCAM), with 1.65 degree diameter field of view, and 67 Mpixels giving $0.6 \mathrm{deg}^{2}$ active pixel area, operating at wavelengths $0.8-2.3 \mu \mathrm{m}$. We provide a short history of the project, and an overview of the technical details of the full system including the optical design, mirrors, telescope structure, IR camera, active optics, enclosure and software. The system includes several innovative design features such as the $f / 1$ primary mirror, the dichroic cold-baffle camera design and the sophisticated wavefront sensing system delivering closed-loop 5-axis alignment of the secondary mirror. We conclude with a summary of the delivered performance, and a short overview of the six ESO public surveys in progress on VISTA.
\end{abstract}

Key words. telescopes - instrumentation: photometers - instrumentation: miscellaneous - instrumentation: detectors

\section{Introduction}

Wide-field imaging surveys have long formed a cornerstone of observational astronomy, from the photographic Schmidt telescope surveys from Palomar, the UK Schmidt Telescope and ESO in the 1950-1980 era. After the advent of large-format CCDs and near-IR detectors during the 1990s, these were followed by a number of major multi-colour digital sky surveys, notably the Sloan Digital Sky Survey (SDSS; Gunn et al. 2006; Abazajian et al. 2009), the 2 Micron All-Sky Survey (2MASS; Skrutskie et al. 2006), the CFHT Legacy Survey (CFHTLS; Cuillandre et al. 2012), and the UK Infrared Deep Sky Survey (UKIDSS; Lawrence et al. 2007). As well as forming a fundamental legacy resource (notably as an atlas for identifications of sources discovered at many other wavelengths, from radio and sub-mm to X-rays and gamma rays), these surveys have led to a very wide range of new discoveries covering most areas and scales of observational astronomy, ranging from asteroids, brown dwarfs, Galactic structure, new Milky Way satellite galaxies, through external galaxies and clusters, out to large-scale structure, weak lensing and the highest redshift quasars.

In late 1998, a new science funding opportunity was provided by the UK Joint Infrastructure Fund; a consortium of 18 UK universities (see Acknowledgements) put together a successful proposal to build a new 4-m class wide-field survey telescope, sited in the southern hemisphere and mainly dedicated to multicolour imaging surveys; this was named the Visible and Infrared Survey Telescope for Astronomy (VISTA). Since 2009, the VISTA telescope and its near-infrared camera (VIRCAM) have been in science operations at ESO's Paranal Observatory: the product of $4 \mathrm{~m}$ aperture and $0.6 \mathrm{deg}^{2}$ on-pixel field of view makes VISTA the world's fastest near-infrared survey system, and it seems likely to retain this advantage until the launch of a dedicated space mission such as Euclid or WFIRST in around 2020.

This paper provides an overview of the development, technical details and on-sky performance of the VISTA telescope and VIRCAM. The intention is to provide an intermediate level of detail across the whole system, plus references to more detailed papers for each subsystem. The remainder of the paper is divided into sections as follows: in Sect. 2 we provide a short outline of the full system, to place in context the more detailed later sections. In Sect. 3 we outline the early history of the project and rationale of the basic design choices; in Sect. 4 we provide an overview of the system optical design and overall layout; Sect. 5 describes the two mirrors and their support systems; Sect. 6 describes the telescope structure and axes rotation systems; Sect. 7 describes the infrared camera; Sect. 8 describes the active optics control; Sect. 9 describes the enclosure and ancillary equipment; Sect. 10 describes computer control and software; Sect. 11 


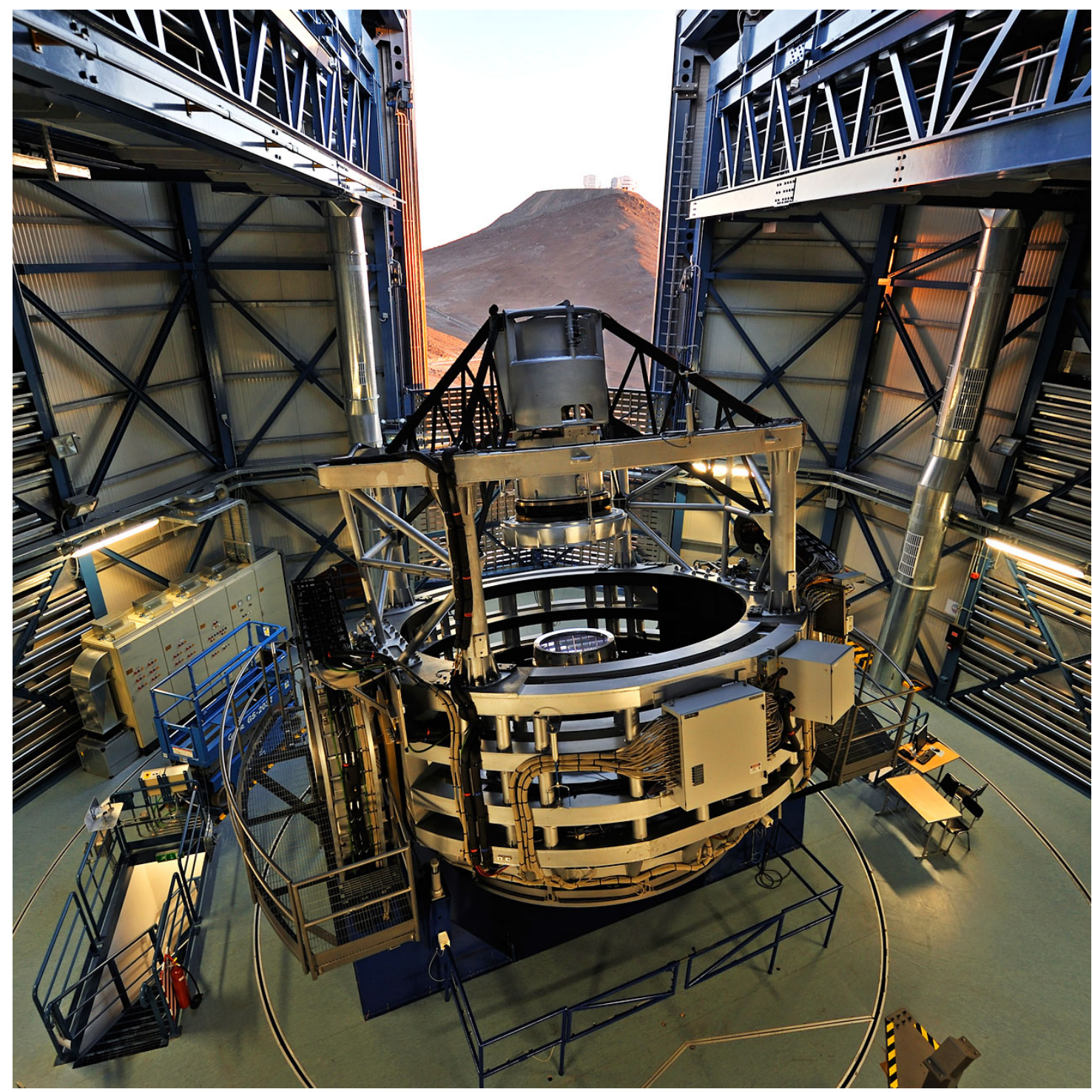

Fig. 1. VISTA telescope at sunset, with the main Paranal summit and VLTs in the background. The VIRCAM vacuum window is visible in the centre of the tube. On the telescope top-end, the M2 hexapod is behind the top ring; the M2 Cell (black) below it, and the M2 Baffle is the metallic annulus. (Photo credit: G.Hudepohl/ESO.)

summarises the commissioning; Sect. 12 summarises observation control, data processing and archiving; and Sect. 13 gives a short summary of the operational performance. These sections are largely self-contained, so the reader interested in particular aspects is advised to read Sect. 2 then skip to the specific section(s) of interest.

\section{System overview}

The VISTA telescope (Fig. 1) is located at ESO's Cerro Paranal Observatory in northern Chile, at latitude $24^{\circ} 36^{\prime} 57^{\prime \prime}$ south, longitude $70^{\circ} 23^{\prime} 51^{\prime \prime}$ west; this is approximately $120 \mathrm{~km}$ south of Antofagasta city, and $12 \mathrm{~km}$ from the Pacific coast. Locally, VISTA is sited on a subsidiary summit of elevation $2518 \mathrm{~m}$, approximately $1.5 \mathrm{~km}$ NNE from the main Paranal summit which hosts the four VLTs, the VLT Interferometer and the VLT Survey Telescope; thus VISTA shares the same outstanding weather conditions. Though VISTA is at approximately $100 \mathrm{~m}$ lower elevation than the main summit, it is rarely downwind of the main summit. Clearly, the southern hemisphere site is very important for complementarity with next-generation major projects including ALMA, SKA Pathfinders and E-ELT, since (apart from 2MASS) the major wide-area optical and near-IR surveys since 2000 (notably SDSS, CFHT Legacy Survey, UKIDSS) are all concentrated in the northern hemisphere.

During routine observing, VISTA is operated entirely in queue-scheduled mode, controlled remotely by a single telescope operator in the main VLT control room; evening startup and morning shutdown procedures are done by an operator adjacent to the telescope for safety reasons.

The telescope optics (Sect. 4) use a very fast two-mirror quasi-Ritchey-Chretien design. The $4.1 \mathrm{~m}$ diameter primary mirror is a hyperboloid of $f / 1.0$ focal ratio; together with a $1.24 \mathrm{~m}$ convex hyperboloid secondary mirror, this produces an $f / 3.25$ Cassegrain focus located behind the M1 cell, $\approx 1.17 \mathrm{~m}$ below the M1 pole (Fig. 2). The Cassegrain is the only focal station, so VISTA carries one large instrument at any time; currently the IR Camera is the only instrument available for VISTA. The telescope is designed for occasional instrument interchange, and a wide-field 2400-fibre multi-object spectrograph (4MOST; de Jong et al. 2012) is approved by ESO for installation after 2019. The telescope uses active (not adaptive) optics; the primary mirror has an 84-point active axial support system, while 


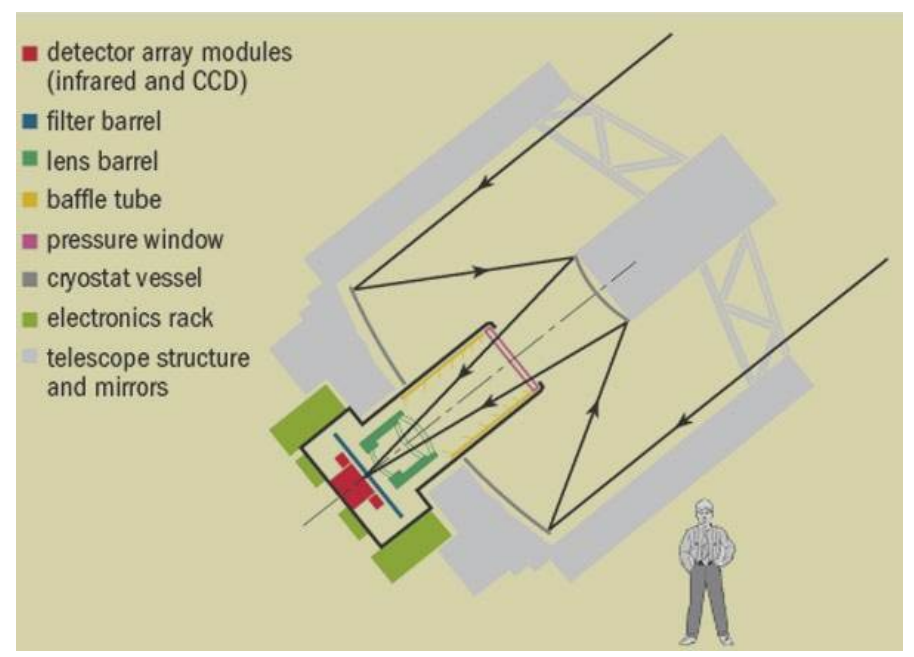

Fig. 2. Schematic view of the general layout of VISTA optical components; this shows the two mirrors, the VIRCAM entrance window and lenses, and the main components of VIRCAM.

the secondary mirror is mounted on a hexapod for 5-axis position control. Physical details of the two mirrors and their support systems are in Sect. 5.

The telescope structure (Sect. 6) is a conventional though very compact Alt-azimuth mount, constructed mainly of steel, with a moving mass of 90 tonnes and sweep radius of $4.6 \mathrm{~m}$. The telescope uses rolling-element bearings on all three rotation axes, and direct position encoding using Heidenhain optical tape encoders with four read heads per axis. The telescope axes are driven via counter-torqued gearboxes for the Azimuth axis and Cassegrain rotator, and two direct-drive motors for the Altitude axis.

The very large VIRCAM (Sect. 7) is mounted on the Cassegrain rotator on the back of the primary mirror cell; the camera mass is 2.9 tonnes including $800 \mathrm{~kg}$ cold, and the cryostat length is $2.8 \mathrm{~m}$. From front to back, the camera includes a $95 \mathrm{~cm}$ diameter vacuum window, a long cold-baffle tube to minimise thermal background on the detectors, a lens barrel with three Infrasil field-corrector lenses, an 8-position filter wheel of $1.37 \mathrm{~m}$ diameter, and sixteen Raytheon VIRGO $2048^{2} \mathrm{HgCdTe}$ near-infrared detectors, giving a mean pixel scale of 0.339 arcsec. The detectors are arranged in a sparse-filled $4 \times 4$ rectangular grid within the 1.65 degree $(350 \mathrm{~mm})$ diameter field of view, and provide an active field of $0.60 \mathrm{deg}^{2}$ on pixels.

The VIRCAM also includes two fixed autoguiders, two fixed low-order wavefront sensors (using CCDs), and movable beamsplitters feeding the science detectors for high-order wavefront sensing, to provide guiding and active optics corrections respectively; details are provided in Sect. 8.

The telescope is housed in a $19 \mathrm{~m}$ diameter enclosure (details in Sect. 9), which follows standard modern design practice to minimise local seeing effects. Features include a powerful aircooling system to maintain the interior at nighttime temperature during the day, active cooling of all electronic boxes in the dome, six large ventilation doors and a movable windscreen to optimise airflow during observing, and a movable moonscreen for reduction of stray light. A single-storey auxiliary building, adjoining the main enclosure on the south side, houses infrastructure facilities including the mirror coating plant, electrical distribution units and glycol pumps.
The system is designed mainly for efficient surveying of large areas of sky: clearly the main design drivers for this are the very wide field of view, moderately large aperture, and the high QE of the detectors; other important contributing factors are the high fraction $\geq 75 \%$ of observing time dedicated to large-scale survey programs, and the minimisation of times for necessary observing overheads, e.g. detector readout and telescope jitter movements. An overview of the on-sky performance is given in Sect. 13.

\section{Basic requirements and design selection}

\subsection{Top-level specifications}

At the start of the project "Phase A" design in April 2000, the basic requirements were as follows. The telescope aperture was set to approximately $4 \mathrm{~m}$ for budget and schedule reasons, availability of an existing mirror blank, and also because achieving a wide field of view becomes progressively more challenging on larger telescopes. The initial baseline was to accommodate two cameras: a $0.3 \mathrm{deg}^{2} 36 \mathrm{Mpixel}$ (9 detector) near-infrared camera, and a $2 \operatorname{deg}^{2} 450$ Mpixel visible camera, both with image quality commensurate with seeing-limited images at a top-class ground-based site. A key goal was to permit an upgrade path for the IR Camera to 67 Mpix and 16 detectors: this upgrade subsequently came to reality, using additional funding provided by the UK, as part of the in-kind contributions for UK entry into ESO membership.

The site was required to be in the southern hemisphere, since all the leading wide-field imaging systems then operating (SDSS) or under construction (CFHT-Megacam, SubaruSuprimeCam, UKIRT-WFCAM) were sited in the northern hemisphere. At the time of VISTA commissioning the only wide-area optical/near-IR southern surveys were photographic Schmidt plates and the 2MASS survey; subsequently the Skymapper (Keller et al. 2007), VST (Cappacioli \& Schipani 2011) and DECam (Flaugher et al. 2012) instruments have begun southern surveys at visible wavelengths which are important complements to VISTA. The site was chosen to be Cerro Paranal in early 2000, and this proved later to be an important step in the UK's subsequent joining of ESO in 2001-2.

The original proposal included two cameras, infrared and visible, with the infrared camera taking precedence in tradeoffs. The proposed visible camera was eventually not built for a combination of several reasons: it was initially postponed due to funding constraints; and the UK's entry to ESO included access to the $2.6 \mathrm{~m}$ VST survey telescope, predicted to be operational earlier than VISTA. However, capability for a visible camera with field diameter $\geq 2.1$ degrees was fully preserved in the telescope design.

The general concept was that the telescope was designed exclusively as the front-end to the IR and visible cameras, and thus general purpose facilities such as additional foci, night-time instrument changes or mid-infrared operations were given zero weight to constrain cost. Spectroscopic capability was also not required, though the final telescope design turned out to be very suitable for a fibre-fed multi-object spectrograph, in particular 4MOST (see Sect. 13).

One key design parameter was the pixel scale in arcseconds: early in the Phase A study, the Science Committee converged on required pixel scales close to 0.33 arcsec for infrared imaging, and 0.25 arcsec for visible imaging, with 10 percent tolerance. The rationale for larger near-IR pixels is due to cost: although atmospheric seeing is slightly better in the near-IR, the 
cost per pixel of near-IR detectors is $\sim 20 \times$ higher than visible CCDs due to the much greater manufacturing complexity and smaller commercial market. Therefore, whereas for a visible imager it is generally affordable to tile the entire correctable field with CCD detectors, this is not true in the near-IR; so the tradeoff between sky coverage and sampling favours coarser sampling in the near-IR than the visible. At that time, standard pixel sizes were $13-15 \mu \mathrm{m}$ for CCDs and 18-20 $\mu \mathrm{m}$ for near-IR detectors, and there was a large cost penalty for non-standard pixel sizes: thus, a physical plate scale near $60 \mu \mathrm{m} /$ arcsec was required for both cameras, which translates to a final focal length near $12 \mathrm{~m}$.

Another key design requirement was for the infrared imager to operate efficiently from $Y$ to $K_{\mathrm{s}}$ bands, i.e. long wavelength cutoff $\geq 2.3 \mu \mathrm{m}^{1}$. Operating at $K_{\mathrm{s}}$ band does add significant challenges, since a room-temperature black surface has $\sim 10 \times$ the surface brightness of the night sky at $K_{\mathrm{s}}$ band; thus, the detectors must be blocked from viewing any significant solid angle of warm high-emissivity surfaces.

Also, an IR camera requires at least a transmissive vacuum window, and typically several transmissive corrector elements. All standard optical glasses absorb strongly beyond wavelength $\lambda>2 \mu \mathrm{m}$, so more exotic materials are required: as the required diameter grows beyond $300 \mathrm{~mm}$, the list of available IR-transmitting materials rapidly shrinks, leading to substantial challenges keeping chromatic aberrations acceptably small with a large physical focal plane. This challenge was overcome using a novel cold-baffle solution, as described later in Sect. 7.

\subsection{Design shortlist}

We here outline some potential concepts which were studied during the early Phase-A studies, before downselecting to the final design with $f / 1.0$ primary mirror and $f / 3.25$ Cassegrain focus.

The initial JIF proposal was for a $4 \mathrm{~m} f / 2$ primary mirror with a flipping top-end and two cameras: a visible camera with corrector lenses using the prime focus, and an $f / 6$ secondary mirror feeding a re-imaging near-IR camera at Cassegrain focus (similar to a larger-diameter version of UKIRT WFCAM; Casali et al. 2007). During the Phase-A study, this evolved slightly to $\approx f / 2.5$ primary, to provide the necessary prime-focus scale for the visible camera with a system $f$-ratio $\approx f / 2.8$ after the widefield corrector. After some investigation, the flipping top-end concept was discarded as unreasonably massive, and a variant was studied with two interchangeable top-end rings, carrying respectively the visible camera and the IR secondary mirror. This concept was feasible, but required a fairly tall telescope structure and large dome, with geometry similar to the William Herschel Telescope. Also, the WFCAM-like layout for the IR camera requires the detector package to be mounted downward-facing, in the beam above the cryogenic tertiary mirror. The resulting tradeoff between tertiary size and central obstruction fraction would make it impractical to expand the field of view beyond $\sim 1.2$ degree diameter, so this route could not reasonably accommodate an upgrade of the near-IR camera to the goal of 67 Mpixels.

Refractive IR camera designs such as CFHT-WIRCAM (Puget et al. 2004) and KPNO-NEWFIRM (Probst et al. 2008)

\footnotetext{
1 We did not consider operation at $\lambda>2.5 \mu \mathrm{m}$, since the Spitzer spacecraft was then in an advanced stage of development, and at $\lambda>3 \mu \mathrm{m}$ the foreground advantage in space is overwhelmingly large. This has proved the correct decision given the excellent performance of Spitzer and more recently the WISE mission.
}

are more compact, but generally require exotic lens materials so again do not readily scale up to fields $>1$ degree.

In parallel with the $f / 2.5$ baseline, other concepts were investigated: two briefly, and one in more detail which was finally adopted in preference to the original. A solution using 2 Nasmyth foci was initially appealing since the planned visibleIR instrument change would be a simple rotation of the tertiary mirror; however, this concept required the focal planes to be well outside the Altitude bearings, in order to avoid pre-focal corrector lenses obstructing the light-path above the primary. This in turn required unreasonably large M2 and M3 mirrors, and very large holes through the altitude bearings, so the Nasmyth option was rejected.

Three-mirror telescope designs have the advantage of excellent image quality over an extremely wide field of view, (e.g. 3.5 degrees diameter for the case of the future LSST; Abell et al. 2009) but there are several penalties: they are significantly more costly for given aperture than 2-mirror systems, have a large central obstruction, and would have great difficulty accomodating a large and massive infrared camera near the top-end. Therefore, 3-mirror designs were also rejected.

In parallel with the original concept, another more compact telescope solution was studied in detail, with an $f / 1.0$ primary mirror and a single $f / 3.25$ Cassegrain focus, feeding interchangeable IR and visible cameras alternately. (We note here that solutions with $\sim f / 1.5$ primary are simple, but could not meet our requirements; the prime focus has too short focal length for an imager, while delivering a $\sim f / 3$ Cassegrain focus behind the M1 would require an unreasonably large secondary mirror).

Compared to the baseline $f / 2.5$ primary, the $f / 1.0$ solution offered lower total cost, reducing moving mass from 250 tonnes to 90 tonnes (Craig et al. 2003) and reducing enclosure size, and enabled a significantly wider near-IR field of view permitting an upgrade path to 16 detectors; so the $f / 1.0$ design was selected at a downselect review mid-way through the Phase A study, and developed thereafter. The final optical design is described in the next section (Table 1).

\section{Optics overview}

\subsection{Optical design}

As described above, the Phase A study selected a novel overall design as follows: the VISTA telescope is a quasi-RitcheyChretien 2-mirror telescope with a very fast $f / 1.0$ primary mirror, and a moderately large $1.24 \mathrm{~m}$ secondary mirror giving a single $f / 3.25$ Cassegrain focal station (Fig. 2); the infrared camera VIRCAM (and the conceptual visible camera) uses this direct Cassegrain focus, with any VISTA instrument including its own wavelength-specific field corrector lenses.

This leads to several unusual features: firstly, the $f / 1.0$ primary mirror (hereafter $\mathrm{M} 1$ ) results in a very compact telescope with a separation of only $2.725 \mathrm{~m}$ from M1 to M2. Also, the infrared camera does not use re-imaging or a cold stop: the $1.24 \mathrm{~m}$ secondary mirror is undersized to form the aperture stop, so each detector pixel views a $3.70 \mathrm{~m}$ off-centre circle on the primary mirror; the envelope of these is a $3.95 \mathrm{~m}$ circle, so no pixel views warm structure outside the primary mirror. The near-IR detectors are located at the corrected telescope focus with no re-imaging optics, and the IR camera includes a long "cold baffle" cylinder extending $2.2 \mathrm{~m}$ above the focal plane to minimise the detectors' view of warm surfaces. (In summary, the IR camera layout is similar to a conventional visible Cassegrain imager, but with the 
Table 1. VISTA: main system parameters.

\begin{tabular}{ll}
\hline \hline Parameter & Value \\
\hline Site & Paranal Observatory, Chile \\
Primary mirror diameter & $4.10 \mathrm{~m}$ \\
Secondary mirror diameter & $1.241 \mathrm{~m}$ \\
Focus & Cassegrain only \\
System focal length (with IR corrector) & $12.072 \mathrm{~m}$ \\
M1-M2 spacing & $2725.7 \mathrm{~mm}$ \\
Mount type & Alt-azimuth \\
Moving mass & 90 tonnes \\
VIRCAM wavelength range & $0.8-2.3 \mu \mathrm{m}$ \\
Field of view (VIRCAM) & 1.65 degree (diameter) \\
Field on IR pixels & 0.6 deg ${ }^{2}$ \\
Mean pixel scale & 0.339 arcsec \\
VIRCAM science detectors & Sixteen Raytheon-VIRGO HgCdTe, 2048 ${ }^{2}$ format \\
VIRCAM guide/wavefront detectors & Six E2V CCD 42-40, 2048 format \\
Filter set (current) & $Z, Y, J, H, K_{\mathrm{s}}$, two narrowbands, one dark. \\
\hline
\end{tabular}

Table 2. VISTA mirror parameters (as-built values).

\begin{tabular}{lcccc}
\hline \hline Mirror & $\begin{array}{c}\text { Physical diameter } \\
(\mathrm{mm})\end{array}$ & $\begin{array}{c}\text { Clear aperture } \\
(\mathrm{mm})\end{array}$ & $\begin{array}{c}\text { Radius of curvature } \\
(\mathrm{mm})\end{array}$ & Conic constant \\
\hline M1 & 4100 & 3960 & 8094.2 & -1.12979 \\
M2 & 1241.5 & 1240.5 & 4018.9 & -5.5494 \\
\hline
\end{tabular}

cryostat greatly expanded to enclose the filter changer, the corrector lens barrel and the primary baffle tube).

The telescope and IR Camera were designed as a single optical system; the conic constants of both mirrors were optimised jointly with the VIRCAM corrector lens surfaces, to optimise image quality averaged over the full 1.65 degree diameter field. The axial thicknesses of the IR Camera window, filters and lenses were included in this optimisation process, but held fixed for mechanical reasons; lens spacings and curvatures were allowed to vary. This leads to mirror conic constants which are slightly different from an exact Ritchey-Chretien system; mirror parameters are given in Table 2. (The visible camera was then separately optimised with these mirror parameters as fixed inputs.)

Dispensing with re-imaging means that the IR Camera corrector lenses only need to correct the off-axis aberrations of the telescope: thus they have weak power, so a single material (Heraeus Infrasil low-OH fused silica) can be used for the vacuum window and all three corrector lenses. Unlike most IR lens materials, Infrasil is robust, highly resistant to thermal shock, very homogeneous, and is available in large diameters.

Thus, the light path is as follows: after reflection from M1 and $\mathrm{M} 2$, the incoming converging beam is transmitted through the flat Infrasil cryostat window, then through three Infrasil corrector lenses, then the passband filter, then finally reaches focus at the IR detector plane.

The 3-lens corrector serves three main functions:

1. To correct the off-axis aberrations (mainly astigmatism) from the 2-mirror telescope.

2. To flatten the focal surface to allow a flat array of detectors.

3. To correct for chromatic aberrations induced by the (flat) vacuum window and filter.

Five of the six lens surfaces are spherical, with the one aspheric surface on the upper (concave) surface of lens 3. The detector plane is flat (allowing slight field curvature was explored, but gave negligible improvement in image quality). The design delivers high throughput and excellent image quality over a
$350 \mathrm{~mm}, 1.65$ degree diameter flat field, without the use of cryogenic mirrors or exotic lens materials.

The image quality on paper (including diffraction with the $1.63 \mathrm{~m}$ diameter M2 baffle, but assuming perfect optical surfaces and perfect alignment) has a $50 \%$ encircled-energy diameter (EED) of $\leq 0.36$ arcsec and an $80 \%$ EED of $\leq 0.68$ arcsec across the entire field of view at $Y$ to $K_{\mathrm{s}}$ bands. Mean values across the field are approximately 0.27 arcsec (50\% EED) and $0.45 \operatorname{arcsec}(80 \%$ EED) with weak passband dependence, leaving a reasonable margin for real-world degradation including optics polishing and support errors, optics misalignments, telescope tracking errors and local dome seeing.

The design does produce significant cubic (pincushion) distortion; the image radius/angle relation is well approximated by

$r(\theta)=12072 \mathrm{~mm}\left(\theta+44 \theta^{3}-10300 \theta^{5}\right)$

where $\theta$ is the off-axis angle in radians. This implies that objects at the corners of the field are imaged at a radius $\approx 0.8$ percent larger than the linear term; thus the (radial) pixel scale $1 /(\mathrm{d} r / \mathrm{d} \theta)$ varies by 2.4 percent from centre to corner of the field. Pixel solid angles are proportional to $(\sin \theta / r) \mathrm{d} \theta / \mathrm{d} r$, and are thus 3.2 percent smaller at the corners of the field. This distortion has no effect on image quality, but needs to be accounted for in image processing: there are two effects, firstly a jitter offset of e.g. 10 arcsec does not correspond to the same number of pixels at all points in the field. Secondly, flat-fielding images to produce the standard convention of uniform counts per pixel in the sky results in photometric variations proportional to pixel solid angles. Both these effects are compensated in the data processing software.

The science filters are placed close to the detector plane (with a nominal clearance of $15 \mathrm{~mm}$ ). This spacing was minimised for two reasons: firstly, to allow location of the autoguider and wavefront sensor units above the filter wheel to minimise electromagnetic or thermal interference between CCDs and IR detectors; and secondly to allow the use of "mosaiced" filters, one pane of glass per detector, without vignetting of detectors by the filter 


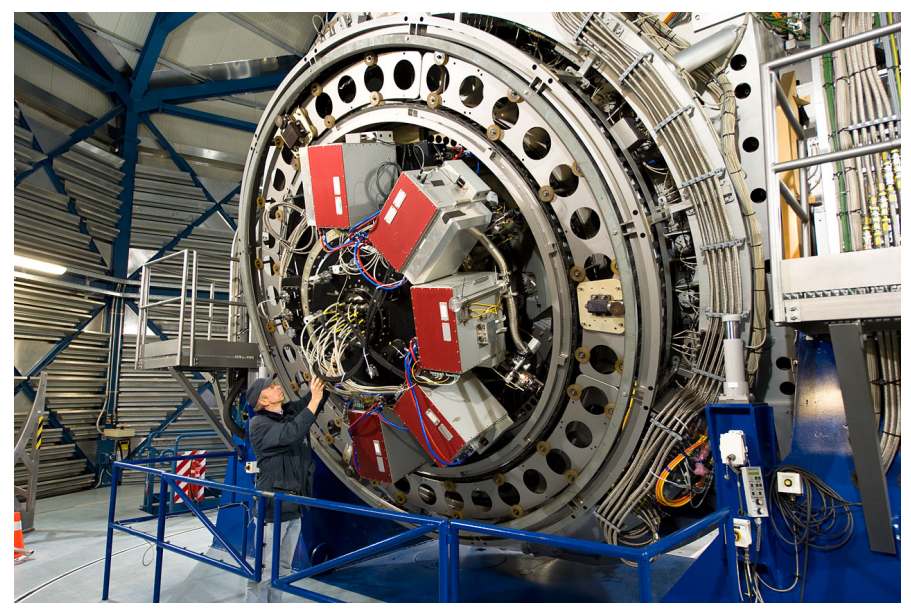

Fig. 3. Back-end of VISTA, with the VIRCAM at Cassegrain focus. The rear of the cryostat is seen (black), with the filter wheel bulge at the far side. The five red electronics boxes are mounted on the cryostat. The camera coolers and cryo-pumps are mostly hidden behind the electronics boxes. The large annular structure surrounding the camera is the Cassegrain cable-wrap; fixed sections of Helium hoses are seen curving around the back of the M1 Cell, and enter the cable-wrap at the connector box at lower right.

support frame, since monolithic filters of the required size and quality would be extremely challenging or impossible to manufacture. The filters operate in the converging $f / 3.25$ beam, with resulting angles of incidence up to 10 degrees. This implies that there is a slight passband smearing since interference filters have a slight dependence of passband on angle of incidence; the effect is nearly negligible for broadband filters, but implies that narrowband filters cannot be much narrower than $1.5 \%$ bandpass.

The design has slight longitudinal chromatic aberration, which is compensated by tuning the filter thicknesses per band to compensate: we chose to fix the $\mathrm{H}$ filter parfocal to $10.00 \mathrm{~mm}$ of fused silica; the M2 position was held fixed across all passbands, while the filter thickness was allowed to vary for other bands for optimal focus. The result is that the $Y$ filter is parfocal to $9.65 \mathrm{~mm}$ silica, while the $K_{\mathrm{s}}$ filter is $10.37 \mathrm{~mm}$ silica. We chose not to allow refocusing the secondary mirror between passbands, because the autoguiders and wavefront sensors use fixed filters selecting roughly $I$-band light, which is folded by a pickoff mirror above the science filter: and refocussing M2 between passbands would have slightly degraded their images and added software complications.

Lateral chromatic aberration is small but not negligible, with approximately 1 pixel shift between the model $J$ and $K_{\mathrm{s}}$ image centroids at the corner of the field. The colour-dependent astrometric shift within any single passband is clearly much smaller, but this will need to be corrected for in the highest-precision astrometry.

The VIRCAM focal plane is located $1172.6 \mathrm{~mm}$ below the pole of the primary mirror, while the Cassegrain rotator back face is $725 \mathrm{~mm}$ below the primary pole: this gives $447.6 \mathrm{~mm}$ back focal distance from the Cassegrain rotator to the detector plane, which is sufficient to accommodate the large filter wheel bulge and several cryocoolers behind the M1 cell structure (Fig. 3).

\subsection{Baffles, stray light, and ghosts}

Baffling against unwanted light-paths from sky to detectors is provided largely by two baffles: the cold baffle tube inside the camera cryostat, and the warm "Narcissus" baffle around M2. The cold baffle has a front aperture of $812 \mathrm{~mm}$ diameter at height $2179 \mathrm{~mm}$ above the detector plane. The warm Narcissus baffle around M2 is a polished aluminium annular baffle of $1.63 \mathrm{~m}$ outer diameter, made as two concave nested spherical surfaces. The curvatures of these spheres are chosen so that the detectors view mainly the black walls of the IR Camera lens barrel reflected in the M2 Baffle. The cold baffle and M2 baffle in conjunction block all direct rays from sky or dome to the detectors. The cold-baffle front aperture is slightly "undersized" and introduces a very small amount of vignetting at the corners of the field of view: the vignetting is zero at angles $\leq 0.69 \mathrm{deg}$, rising to $1 \%$ at the corner of the field. This small amount of vignetting was an intentional choice, since the undersizing of the cold baffle reduces the required $\mathrm{M} 2$ Baffle diameter compared to a strict zero-vignetting system, and this reduction produces benefits for diffraction and overall average throughput.

Given the absence of an intermediate cold stop, stray light and ghosting were a significant concern (Patterson \& Wells 2003), and careful attention was paid to minimising these by design: all surfaces around the light beam are shaped to minimise first-order scattered light, using various features such as ridges on the spider vanes, grooved walls in the camera lens barrel, chamfered edges of the filter mount trays, etc. The VIRCAM corrector lenses are generously oversized with a physical radius at least $2 \mathrm{~cm}$ larger than their useful aperture; this keeps the lens edges "in shadow" behind support structure, avoiding stray reflections from the lens outer edges. Additional baffling against moonlight illuminating the optics is provided by a movable moonscreen at the top of the slit (Sect. 9).

Ghost images from unwanted reflections are present (as in any transmissive system); in VIRCAM the dominant ghosts are the localised doughnut-shaped out of focus ghosts from unwanted reflection at the filter. The smallest ghost is from light reflected twice inside the filter; brighter stars $(J \lesssim 7)$ also show two larger and fainter ghosts, arising from one reflection off the detector surface followed by a second reflection from the bottom or top of the filter. These three ghosts are $\approx 63,144$ and 205 arcsec in diameter respectively. These filter ghosts were essentially unavoidable, given the several optomechanical constraints requiring the filters to be located close to the detector plane; the ghosts are localised around the main star image (though not precisely concentric), and are easy to recognise in the images. To date, very few "non-local" ghosts have been detected $^{2}$, and there is no significant "sky ghost" or "pupil concentration" effect, as can arise from reflections between detector and corrector lenses.

\subsection{No-lens test setup}

We note that for testing the telescope without VIRCAM, using the 2 mirrors alone without the VIRCAM field corrector (with unchanged M1-M2 spacing) produces unacceptable spherical aberration at the resulting bare focus. However, a good "bare telescope" configuration with no lenses can be found by allowing both the M2 and focus locations to vary. The solution is to move M2 downward by $2.1 \mathrm{~mm}$ with the hexapod, and locate the detector at the new focus $1153.2 \mathrm{~mm}$ below M1 pole. This setup corrects both defocus and 3rd order spherical aberration, and gives a usable field of view $\sim 5$ arcmin diameter, limited by residual off-axis coma since the telescope is not an exact Ritchey-Chretien. The 5 arcmin field is ample for test purposes,

\footnotetext{
2 There is one suspected ghost $\sim 3$ degrees from the very bright star Mira Ceti.
} 
and was used for the initial telescope commissioning phase prior to first light of VIRCAM. In practice, we set up the test camera location by metrology with respect to M1, then moved M2 to best focus, and verified that the spherical aberration was minimal as predicted.

\subsection{Optical design summary}

As above, the overall system design is strongly optimised for wide-field survey operation with infrequent instrument changes. The design has many beneficial features: the telescope structure is very compact and rigid, leading to a small enclosure, reduced costs, fast jitter movements and minimal windshake. The coldbaffle camera design enables a very wide field of view with good image quality across $Z$ through $K_{\mathrm{s}}$ bands, excellent throughput and no exotic lens materials; and the Cassegrain instrument station can readily accommodate the large and massive VIRCAM and associated service bundles.

The telescope also offers good potential for future instrument(s): the large Cassegrain volume implies that the potential field of view is limited mainly by manufacturability of the new field-corrector, rather than telescope constraints. For the planned 4MOST instrument, corrector designs up to 3 degrees diameter were developed in the concept phase; after cost/benefit tradeoffs the 4MOST baseline is currently 2.5 degrees (de Jong et al. 2012, see also Sect. 13).

The main downsides of the design are that the fast $f / 1$ primary mirror is highly aspheric, and was even more challenging than expected to figure to the required accuracy, leading to unanticipated delays in project completion. Also the relative alignment tolerances of $\mathrm{M} 1 / \mathrm{M} 2$ are very stringent, which is solved using active optics as detailed in Sect. 8. Finally, the camera vacuum window is very large and gave challenges with manufacturing and thermal design, as in Sect. 7.

\section{Mirrors and mirror support}

In this section we summarise the physical details of the two mirrors, and their support systems including the M1 active force actuators and the M2 Hexapod. Where needed, we refer to a coordinate system in which $+z$ is parallel to the telescope optical axis, $x$ is parallel to the telescope altitude axis, and $y$ is perpendicular to both, i.e. the downward tangent to the primary mirror.

\subsection{Primary mirror}

The primary mirror (Fig. 4) is made of Zerodur glass-ceramic, with the blank manufactured by Schott Glas, Germany; a preexisting thicker Zerodur blank was machined down by Schott Glas to the final meniscus (Doehring et al. 2004). The mirror geometry is a solid meniscus, of $4.10 \mathrm{~m}$ outer diameter with a $1.20 \mathrm{~m}$ diameter central hole, a thickness of $17 \mathrm{~cm}$, a spherical back surface, and a mass of 5.5 tonnes including the support pads. The measured coefficient of thermal expansion (CTE) is $0.066 \mathrm{ppm} / \mathrm{K}$, small enough that thermal effects on the figure during operations are negligible.

The mirror optical surface was polished by LZOS, Moscow, (Abdulkadyrov et al. 2008a) to the required hyperboloid figure as in Table 2. This was a challenging and time-consuming process due to the high asphericity of the fast mirror (deviation over $800 \mu \mathrm{m}$ from the best-fit sphere); but was eventually completed to good quality with an rms wavefront error of $35 \mathrm{~nm}$ (after theoretical subtraction of available low-order active force patterns).

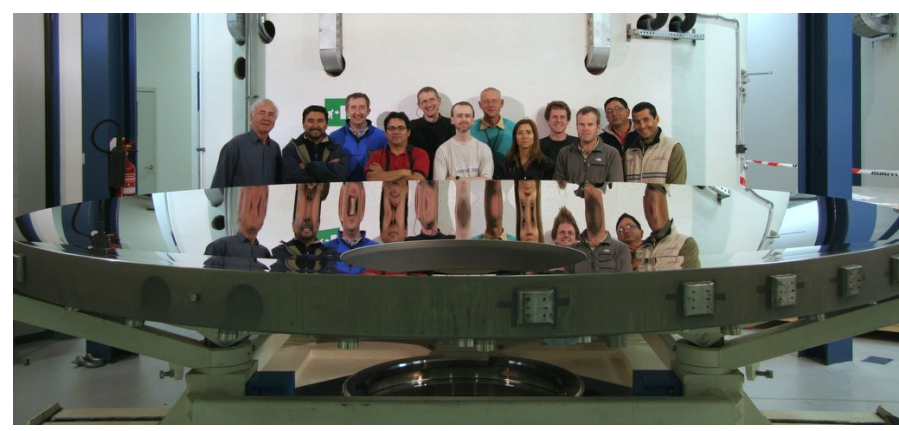

Fig. 4. VISTA primary mirror on its wash-stand, after the first coating in April 2008. The telescope pier is behind the group. The apparent "kink" near the front arises from two reflections off the mirror.

The figuring process involved numerous cycles of wavefront measurement and polishing, roughly once per week for the two year figuring process. Wavefront measurements sometimes used two independent null correctors of differing design, to guard against the well-known HST-type disaster arising from a manufacturing error in a single null corrector. In addition, one of the null correctors was independently validated using a purposemade computer generated hologram (CGH).

Mirror coating (silver or aluminium) is described in Sect. 9.

\subsection{M1 supports}

During observations, the M1 is supported against gravity and wind loads by numerous pneumatic force actuators which deliver controlled forces to the mirror back and sides, and it is held in position relative to the Cell by six definers. More details of this system are given by Stobie et al. (2010).

The definers are quasi-rigid metal cylinders with 2-plane flexure joints at both ends, so each definer provides essentially a one-dimensional position constraint: thus the six definers combined provide kinematic location of the M1 relative to the Cell, without overconstraint stresses from flexure of the Cell. Each definer's axial stiffness is $30 \mathrm{~N} \mu \mathrm{m}^{-1}$, which leads to M1 rigidbody rocking frequency above $15 \mathrm{~Hz}$. Each definer incorporates a load-cell measuring the force component along its long axis: these force readings are used as the inputs to a fast servo loop controlling the pneumatic mirror supports, to keep the residual forces on the definers at desired values (see later).

There are three axial definers (parallel to the $z$-axis) equispaced around the back of M1, and three lateral definers attached tangent to the M1 back near the outer edge. All the definers include force-limiting spring-plate devices, so they "break away" and become spring-loaded at axial force exceeding $1200 \mathrm{~N}$; this avoids excessive point loads on the mirror and load cells when the pneumatic supports are turned off, or faulty, or in the event of an earthquake.

Each of the six definers includes a precision lengthadjustment screw and dial giving $1 \mathrm{~mm}$ length change per revolution: these are accessible by hand from behind the M1 Cell, enabling the M1 position to be adjusted to $\sim 25 \mu \mathrm{m}$ resolution. This is essentially a set-and-forget adjustment to align the M1 to the Cassegrain rotator axis, since the Cell structure is designed to deliver minimal flexure between the M1, Cassegrain rotator and Camera (see Sect. 8 for more details). Six linear variable displacement transducers (LVDTs) provide monitoring of the M1 position relative to the Cell to $\approx 2 \mu \mathrm{m}$ resolution; this position information is not used in closed-loop, but provides a health-check on the support system, and also verifies 


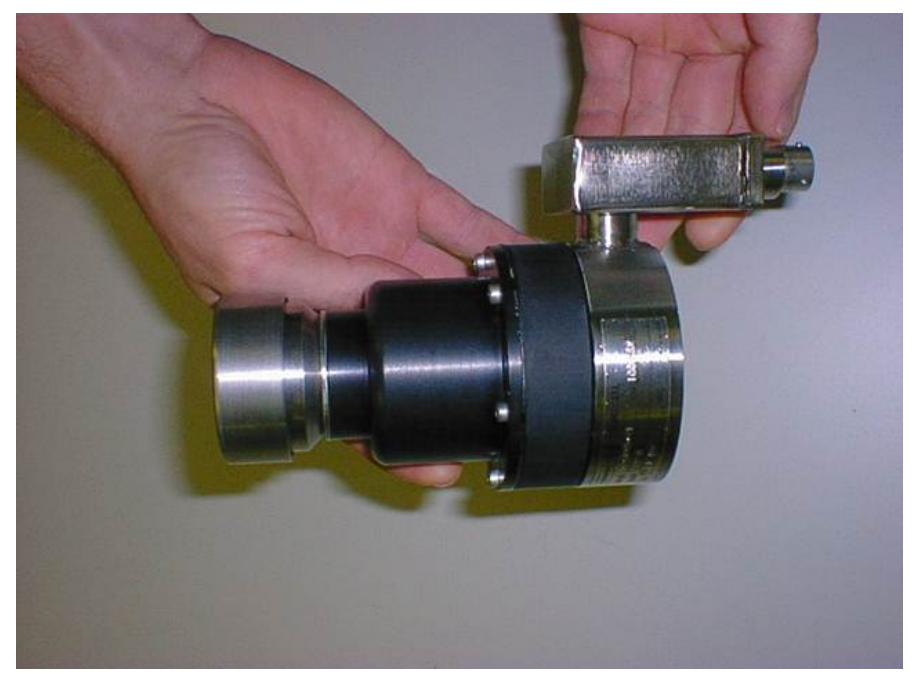

Fig. 5. One of the 81 primary mirror axial supports. In operation, the metallic cap (at left) pushes against the M1 metal pad via the pneumatic cylinder at centre. The load-cell is on the right, with readout electronics in the cuboid unit above.

that any desired manual alignment adjustments have been applied correctly.

The M1 supports interface to the mirror via super-Invar pads glued to its back and side: there are 84 axial support pads on the back surface, located on four circles of 12, 18, 24, 30 supports respectively, giving a six-fold symmetry in the pattern. There are 24 lateral support pads around the outer cylindrical face. A further 3 pads on the back face (near the outer edge) provide attachment for the lateral definers. For the axial supports, a stainless steel wedge is bolted to each Invar pad to provide a flat surface perpendicular to the $z$-axis, against which the axial support piston pushes.

All the M1 force actuators are pneumatic pistons containing a Bellofram membrane (Bennett \& Baine 2004): there are 81 identical axial actuators (Fig. 5), each with its own pressurecontrol valve and load cell, thus all the axial support forces are controllable individually by software. The axial supports are "push only" with an operating force range of $5 \mathrm{~N}-990 \mathrm{~N}$, compared to the mean gravity load of $645 \mathrm{~N} \sin$ (Alt) per support, and $\mathrm{rms}$ force accuracy $\approx 1 \mathrm{~N}$. The air pressure for each support is controlled in closed-loop using the load cell value, and load cell readings are reported periodically to the telescope control system for automatic status checking. Active optics "pull" forces are simply delivered by pushing less than the mean gravity load, so this limits the available active force range to approximately $\pm 250 \mathrm{~N}$ per support.

The remaining 3 points in the 84-point axial support pattern are the passive axial definers: the fast-balancing servo loop reads the definer load forces as input, and adds piston and $x / y$ gradient modes to the 81 axial supports in order to keep the definer loads at their fair share of the overall load. This force balancing loop runs fast, at $\approx 20 \mathrm{~Hz}$ framerate, to ensure that varying wind and gravity loads are rapidly shared out across all the supports, avoiding residuals at the axial definers. The active force pattern adjusting the M1 figure (Sect. 8) is additive to the balancing forces, but this loop runs much more slowly, updating approximately once per minute during telescope tracking. In the event that one axial support is defective, the software can disable it and use pre-computed lookup tables to re-distribute its force across the remaining supports with minimal effect on the M1 figure.
The M1 lateral supports are also pneumatic, and comprise twelve "push only" supports below the mirror, and twelve "pull only" supports above the mirror. The lateral supports are tilted up/down with varying angles (roughly tangents to the mirror surface) optimised by finite-element analysis, but all act along lines perpendicular to the telescope Altitude axis in the $y z$ plane, i.e. there are no force components parallel to the Altitude axis. The lateral supports are not controlled individually, but are fed by four pressure-control valves each feeding one quadrant of six supports: currently all four valves receive one common pressure demand, but the use of four parallel valves provides faster response. The lateral force control loop simply servos the pressure demand so that the average of the forces measured by the 4 o'clock and 8 o'clock lateral definers is zero; thus the lateral supports balance the component of gravity load (and wind, if any) in the tube $y$-direction. Any small $x$-forces or $z$-moments (resulting from deviations from the nominally symmetrical system) are reacted passively by the lateral definers; these residual uncontrolled forces can be checked to be small using the lateral definer load readings.

\subsection{M1 rest-pads}

In addition to the active pneumatic support system, the M1 Cell contains a system of 18 passive rest-pads which support the M1 when the support system is turned off, or the telescope is parked, or in an earthquake condition. The rest-pad system comprises twelve axial rest-pads (in two rings of six) behind the M1, and six lateral rest-pads equispaced around the side of M1. Also, above the mirror there is a failsafe "restraint clamp" which can press down on the periphery of the M1 front surface (outside the optically useful area).

The rest pad positions were set as a one-time adjustment so that each pad has a nominal $1 \mathrm{~mm}$ air gap to the M1 back or side face when M1 is at its operational position. Each rest pad comprises a plastic-foam-steel sandwich, with the plastic closest to M1. The $3 \mathrm{~mm}$ thick foam layer provides shock absorbing in the event that M1 strikes the rest pads at significant speed, which can happen in cases of an earthquake, or a major software fault "dropping" the mirror.

The restraint clamp is a rubber-coated steel ring covering the outermost $3 \mathrm{~cm}$ of the M1 upper surface (which is unseen by the VIRCAM detectors). The clamp has a failsafe design: in the power-off or parked condition, the clamp is pressed downwards on the M1 by sixteen permanent springs; during observations, the clamp ring is retracted upwards above M1 by compressed air pistons which overcome the spring forces. When the telescope Altitude is below 20.5 degrees, or if the earthquake sensor trips, the clamp air supply is cut off by hardware, and the springloaded clamp presses M1 against the axial rest-pads with $\approx 24 \mathrm{kN}$ downforce.

A deliberate omission is that VISTA has no on-telescope M1 cover. This is because the tube and camera design do not allow space for a "concertina" design (and these are also prone to jamming), while "petal" designs were rejected due to degrading the airflow across the primary mirror when open. Various precautions are followed for M1 safety and minimisation of dust buildup: during the daytime the telescope is normally parked at Altitude $=20$ degrees south, with the M1 clamp applied. A commercial earthquake sensor inside the pier also applies the clamp if an earthquake is detected. A hardware interlock prevents the slit doors moving open unless the telescope is in this park position and the moonscreen is deployed (this does not apply to door closing). All lifting operations except M1 removal 


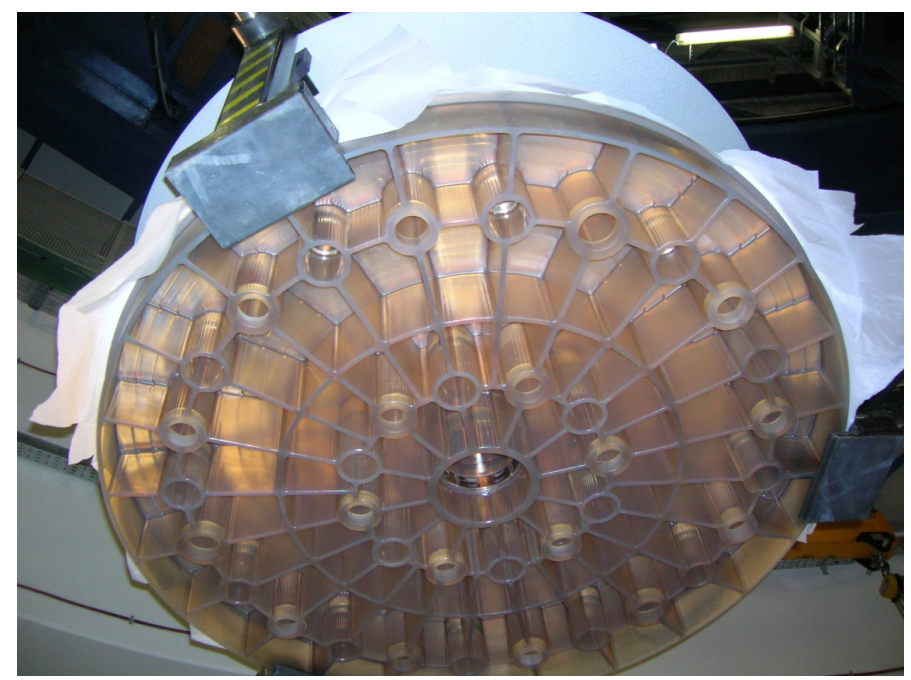

Fig. 6. Rear view of the VISTA secondary mirror, showing the lightweighting pattern. The astatic lever supports interface to the cylindrical sockets.

are performed with the telescope horizon-pointing; an external M1 cover is used during removal of M1 from the telescope.

\subsection{Secondary mirror}

The convex secondary mirror (M2) is made of Astrosital ultralow expansion glass-ceramic; optical parameters are in Table 2. Both the M2 blank manufacture and the optical polishing (Abdulkadyrov et al. 2008b) were done by LZOS, Moscow, as for the M1 polishing.

The M2 has a diameter of $1241.5 \mathrm{~mm}$, central thickness $156 \mathrm{~mm}$ and is approximately $70 \%$ lightweighted, giving a mirror mass of $113 \mathrm{~kg}$; this lightweighting was achieved by several months of careful machining starting from a solid block of Astrosital. The M2 face-plate is $\approx 25 \mathrm{~mm}$ thick, and has an overhang of $10 \mathrm{~mm}$ at the outer rim so that the annular baffle surrounding M2 can overlap behind it, avoiding detectors viewing sky through a gap. Since the M2 forms the aperture stop for the optical system, the full diameter of M2 is used optically, except for a $0.5 \mathrm{~mm}$ wide bevelled edge around the circumference and a flat patch in the centre used as a coarse-alignment target during initial setup at Paranal.

The M2 optical testing used a purpose-made Hindle sphere of $2.4 \mathrm{~m}$ diameter, used with the M2 downward-facing supported on the same Cell as used in operations.

The M2 is supported from a $\mathrm{MgAl}$ alloy Cell containing a passive 36-point astatic lever system. The M2 lightweighting pattern (Fig. 6) includes 36 hollow cylindrical pockets machined into its back face, in two rings of 12 and 24 respectively; these pockets are alternating between axial and lateral supports. Each pocket has a metal rod attached which passes through a matching hole in the M2 Cell. All the 18 lateral supports, and 15 of the axial supports have a lever-and-counterweight arrangement, with the pivot point attached to the Cell and the counterweight inside the Cell; the lateral supports use a straight rod and hinge, while axial supports use an inverted-L arrangement; by basic geometry, these levers deliver lateral and axial forces on the mirror which vary as the desired cos or sin function of altitude (modulo friction). The remaining 3 axial support points are quasi-rigid rods to provide rigid positioning of the M2 position relative to the Cell. Lateral centration is provided by a large boss on the

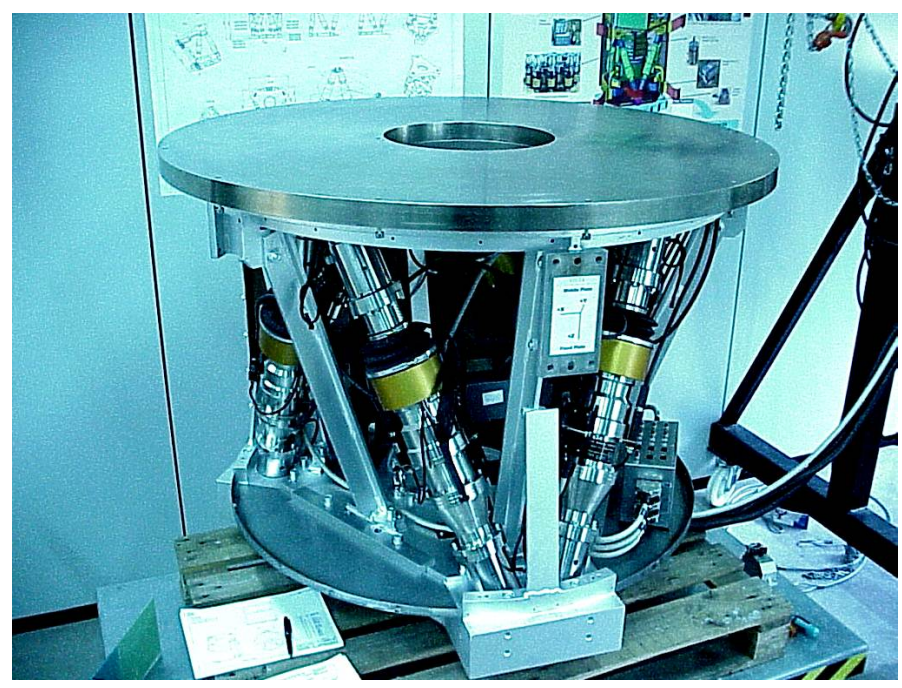

Fig. 7. VISTA M2 Hexapod positioner during testing at NTE, Barcelona. Here the hexapod is inverted from its operating orientation, so the mobile plate is on top.

middle of the Cell with rollers which slide in a matching socket at the centre of the M2 back.

\subsection{Hexapod}

In the telescope, the M2 and its Cell are both supported by a large high-precision hexapod: its purpose is to adjust the position of the $230 \mathrm{~kg} \mathrm{M} 2+$ Cell assembly in 5 axes (focus, centering and tilt) to a differential step accuracy of $\approx 1 \mu \mathrm{m}$ and 0.1 arcsec, using information from the wavefront sensors (Sect. 8), in order to keep the optical system precisely focused and collimated under the varying thermal expansion and gravity loads on the telescope tube.

The hexapod (Fig. 7) was manufactured by NTE, Barcelona (Geijo et al. 2006), and is a close relation of that used in the Gran Telescopio Canarias $10 \mathrm{~m}$ telescope, with the fast-chopper stage eliminated since VISTA will not observe mid-infrared wavelengths $\geq 2.5 \mu \mathrm{m}$. The hexapod has approximate dimensions of length $90 \mathrm{~cm}$, outer diameter $110 \mathrm{~cm}$ and mass of $600 \mathrm{~kg}$.

The correction demands to the hexapod are slow, typically updating once per minute. Thus, the VISTA system has no highspeed guiding or fast tip-tilt correction: windshake rejection relies on the combination of the rigid telescope structure and the good wind attenuation provided by the enclosure.

As the name implies, the hexapod contains 6 legs with independent length adjustment: these connect the "fixed plate" bolted to the Telescope top-end central barrel to the "mobile plate" which supports the M2 Cell and M2. Each hexapod leg is made in two parts, coupled by a high-precision planetary roller screw of $1 \mathrm{~mm}$ pitch; a servo motor turns the screw to adjust the leg-length. Leg extension is measured in closed-loop by a Heidenhain optical tape linear encoder with resolution $0.1 \mu \mathrm{m}$, while motor control also uses a rotary encoder. The leg-length travel range is $\pm 5.5 \mathrm{~mm}$ to electrical limit switches, and $\pm 8 \mathrm{~mm}$ to mechanical endstops.

The hexapod legs are attached to the fixed and mobile plates via $\mathrm{CuBe}$ alloy flexure rods: these are sized to allow bending at the joints up to $10 \mathrm{mrad}$ as the hexapod moves, while providing highly repeatable motion. Safety cylinders around each flexure joint provide an electrical cutout which stops motion of all the legs in the event of excessive tilt at any joint. An internal wire 


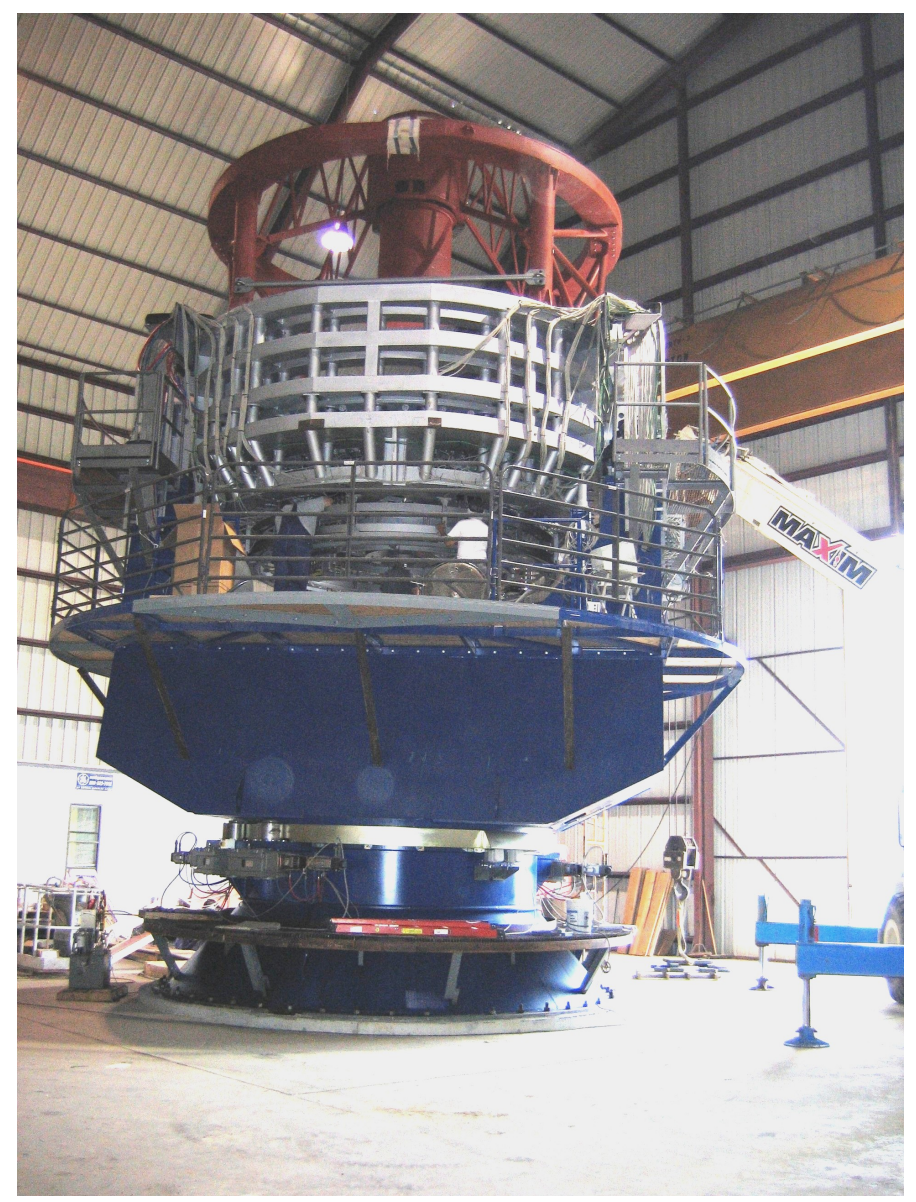

Fig. 8. VISTA telescope structure in 2005, during testing at the Vertex plant in Mexia, Texas.

rope prevents M2 falling in the event of catastrophic failure of flexure $\operatorname{rod}(\mathrm{s})$.

A fixed framework mounted to the hexapod fixed plate outside the legs supports the outer covers and the M2 Baffle, therefore these do not load the hexapod legs and the M2 is well shielded from wind loads.

The hexapod is "slow" with a response time for small offsets approximately $5 \mathrm{~s}$. When small position steps are commanded by the active optics software, the low-level hexapod control software moves the six legs at proportional rates, so that adjustment steps in focus and centration (rotation around M2 centre of curvature) give negligible image shift, and these corrections are applied while science exposures are active. In contrast, M2 tilt corrections do produce image shift, therefore cannot be applied during science exposures: these corrections are buffered by the telescope control software so they are only applied in between consecutive science exposures (see Terrett \& Sutherland 2010).

\section{Telescope structure}

The telescope structure and axis controls (and also the M1 support system) were manufactured by Vertex-RSI (now General Dynamics) of Mexia, Texas; many details are given in Jeffers et al. (2006). Much of the altitude and azimuth bearings and drive systems re-use the design of the $4 \mathrm{~m} \mathrm{SOAR}$ telescope at Cerro Pachon, though the optics and tube structure of VISTA are very different. A view of the complete telescope structure during factory testing is shown in Fig. 8.
The key design aims of the telescope structure were to provide good tracking performance, to maximise stiffness (for windshake rejection), to minimise deflection between the M1 and Camera during operation, to provide fast offsetting for jitter movements during observing, and to provide fault-tolerance and easy maintenance for low operation costs; these have generally been successful.

One notable feature is that the telescope uses rolling-element bearings on all three rotation axes: these have slightly higher friction than the hydrostatic bearings on most modern large telescopes, but offer performance that meets our specifications at lower cost and significantly reduced maintenance, since highpressure oil pumps are not required.

The telescope tube does not use the traditional Serrurier truss principle: instead, to maximise overall stiffness the M1 Cell is rigidly coupled to the central Altitude ring, and the gravitational deflection of the top-end with varying altitude $(\sim 0.4 \mathrm{~mm}$ max $)$ is compensated in closed-loop by the active optics system and the M2 hexapod.

The overall Telescope moving mass is 90 tonnes, including mirrors and VIRCAM: this divides as 44 tonnes in the "tube assembly" rotating around the altitude axis, and 46 tonnes in the azimuth structure. The lowest natural frequency of the structure including the pier is $9.2 \mathrm{~Hz}$, showing good overall stiffness.

The telescope structure comprises various subsystems, as follows.

\subsection{Azimuth rotation system}

The telescope is supported on a cylindrical concrete pier, of height $3.66 \mathrm{~m}$, outer diameter $6.0 \mathrm{~m}$ and inner diameter $4.0 \mathrm{~m}$. The hollow centre of the pier contains the hanging-spiral azimuth cablewrap, accessible via a door at ground level and internal ladders. A central pillar of $1 \mathrm{~m}$ diameter supports the azimuth encoder tape disk above. On top of the pier is the fixed pedestal, a 20 tonne steel cone structure: this pedestal provides a precision flat upper surface supporting the telescope azimuth rotation bearing, and rigid mounts for the azimuth drive gearboxes.

The azimuth structure rotates on a rolling element bearing of $3.66 \mathrm{~m}$ main race diameter. The azimuth rotation is driven by a set of four identical motor and gearbox systems, operated in two counter-torqued pairs to minimise backlash. Each motor (a commercial Kollmorgen DC servomotor) drives a commercial Bayside 70:1 planetary high-speed gearbox, then a custom-made 10.65:1 low-speed right-angle gearbox fixed to the pedestal. Each low-speed gearbox turns a 37-tooth helical pinion which engages with the 728 teeth machined on the outer face of the azimuth bearing ring; thus, the total gear ratio is $14671: 1$, or 88.3 arcsec telescope rotation per motor revolution. This large gear ratio provides fast acceleration and offsetting performance; the maximum slewing speed is $2 \mathrm{deg} / \mathrm{s}$, limited by the $5000 \mathrm{rpm}$ limit of the motors.

The Azimuth bearing supports the yoke, a large U-shaped steel plate structure, of mass $\approx 32$ tonnes, which rotates in azimuth and supports the telescope altitude bearings on its upper faces. Hatches are provided in the yoke for access to the various cables and hoses. The yoke also supports the circular rotating floor forming the central part of the Dome floor, and two side platforms giving easy access to the Altitude drives.

\subsection{Altitude rotation system}

The Altitude rotation system is fully symmetrical, with identical bearings, drive motors and encoders on both sides of the 
telescope tube, to minimise structural torquing effects. The two altitude bearings are large rolling-element bearings; each side uses a pair of preloaded bearings using conical rollers, outer diameter $920 \mathrm{~mm}$. The altitude axles are hollow, enabling safe access to the M1 side supports by removing the motor cover and crawling through the $630 \mathrm{~mm}$ bore. The altitude axis is driven by a pair of identical direct-drive motors adjacent to the bearings.

The altitude axis motion has several layers of safety features to avoid out-of-range collisions: firstly, velocity limit switches constrain the maximum velocity to $10 \%$ of slewing speed as the axis position approaches a hardware limit. Next, pre-interlock switches command the motor drives to back away from the limit under hardware control. Next, interlock switches cut the motor power and apply the failsafe air-brakes. Final protection is provided by two pairs of large oil-filled dampers mounted on the yoke; if the tube travels out of range to Altitude $\approx 92^{\circ}$ or $-3^{\circ}$, the back of the M1 Cell hits one pair of dampers, which are calculated in theory to stop the tube from $4 \mathrm{deg} / \mathrm{s}$ (twice the operational limit) without causing damage. For prevention of overspeed in the event of control failure, a small gyroscope fixed on the tube interlocks the drives if the tube angular speed exceeds $2.5 \mathrm{deg} / \mathrm{s}$.

\subsection{Telescope tube}

The telescope tube assembly, supporting the optics and rotating in altitude, comprises three main parts: the M1 Cell, Altitude ring and Top-end structure. The 15-tonne Altitude ring and 12-tonne M1 Cell were manufactured in separate weldments for ease of transport, but were permanently bolted together during installation on site: these form a rigid "bucket" structure which connects the M1 definers and the Cassegrain rotator to the Altitude rotation axles. Both parts are a lattice structure of steel tubing, to provide improved ventilation of the primary mirror and access to the M1 support system. Since the Cassegrain rotator axis forms the fundamental alignment reference of the system, it is helpful that this is never detached from the body of the telescope.

For optimal alignment, the telescope structure had specific and stringent specifications for the relative deflections between the M1 and the Cassegrain rotator. In order to meet these, a novel structure for the Cell was used: the lower section contains a rigid triangle beam which links the M1 definers to the Cassegrain rotator, while a separate relatively lightweight "basket" in the upper part of the cell carries the M1 axial supports. This means that flexure of the basket is absorbed by length changes in the pneumatic M1 supports (which run in a fast force-control loop), while the passive M1 Definers keep the mirror position quasi-static relative to the triangle beam; this minimises relative deflections between the Camera and the M1, for optimal image quality. Details of the flexure measurements are provided in Jeffers et al. (2006).

The telescope top-end structure is a 6.5 tonne assembly comprising trusses, top-ring, spiders and the central "barrel" structure. The complete top-end structure (without M2) can be detached from the Altitude ring and removed in one piece (horizon-pointing), then stowed on a cradle on the dome floor for M1 removal and recoating (see Sect. 9 for details).

The top-ring has an inner diameter of $4.5 \mathrm{~m}$ (which is sized to not vignette a 4 degree diameter field of view, in case of future instrument enhancements, such as a possible wide-field spectrograph). To maximise stiffness in the focus direction, the spiders are slanted upwards, and are non-radial, joining the central barrel at two opposite points. The spiders (each $36 \mathrm{~mm}$ total width) incorporate several small ridges on their side faces, to minimise grazing-incidence stray light scattering off the spider sides and reaching the detectors.

The top-end central barrel is a passive steel structure, providing a rigid link between the spiders and the Hexapod fixed plate: it has a diameter of $\approx 1.4 \mathrm{~m}$ and mass 1 tonne. The barrel is hidden from the detectors by the $1.63 \mathrm{~m} \mathrm{M} 2$ Baffle below. The barrel has a central hole of $25 \mathrm{~cm}$ diameter, used for early onsky testing with a $20 \mathrm{~cm}$ telescope mounted on the Cassegrain axis. A recess on top of the barrel houses the Hexapod electronics control box; this box is glycol-cooled, via feed hoses running in an insulated channel along the top of one spider leg.

\subsection{Cassegrain rotator}

As for any altazimuth telescope, it is essential to compensate for image rotation around the optical axis as the telescope tracks the sky. The Cassegrain rotator is attached in a recess on the back of the M1 Cell, and forms the main telescope to camera interface: this rotates the 2.9 tonne VIRCAM around its axis. The rotator is driven by one pair of counter-torqued motor and gearbox systems, which are recessed into the M1 Cell and drive a helical gear on the outer face of the Cassegrain rotator. The rotator bearing is a ball-bearing with $1.45 \mathrm{~m}$ race diameter; the central bore is $1.35 \mathrm{~m}$ diameter, to allow the primary mirror lifting tool to pass through during M1 handling.

\subsection{Telescope drives and encoders}

As noted above, the telescope axes are driven via countertorqued gearboxes for Azimuth (four) and Cassegrain (two), while two direct-drive motors drive the Altitude axis. Angular position measurement is provided by Heidenhain ERA $880 \mathrm{C}$ optical tape encoders on all three axes: the Azimuth and Cassegrain axes each use a single full-circular encoder tape with four equispaced read-heads, while the Altitude axis has a pair of semicircular optical tapes on either side of the tube, with two read-heads (fixed to the yoke) at \pm 45 degrees from the vertical on each side.

The encoder tape diameters are respectively $1638 \mathrm{~mm}$, $1854 \mathrm{~mm}$ and $1578 \mathrm{~mm}$ for the azimuth, altitude and Cassegrain axes. The encoder tapes have grooves with 25 lines/mm; each readhead provides "sin" and "cos" signals which are electronically interpolated to 12 bits (4096 counts) per groove, and averaged in the electronics; thus one encoder least-significantbit corresponds to approximately $10 \mathrm{~nm}$ at the tape, or $\sim 0.0025$ arcsec around the axis.

The control system for each axis uses an outer position loop running in the axis LCU (Sect. 10) based on comparing actual and demanded encoder readings; this outputs an analogue velocity demand to the low-level axis control electronics, and in turn this sends current demands to the power amplifiers driving the motors. Since these inner loops are fast and the axis encoders have very high resolution, unwanted effects such as motor torque ripple, gearbox imperfections and backlash are largely eliminated by the servo loops, and the telescope tracking performance is good (see Sect. 6.7 for tracking errors).

\subsection{Cable wraps}

The VISTA cable-wraps are substantial, due mainly to the 10 high-pressure Helium hoses feeding the IR Camera, in addition to many signal cables and glycol coolant hoses.

The Azimuth cable-wrap is a hanging spiral inside the pier, using metal hoops suspended on chains to offload the self-weight 
of the cables and prevent tangling: this wrap is simply pulled around by the telescope drives. The Altitude cable wraps (one on each side, with separate sides for power drives and signals) use Igus energy-chain devices, also pulled by the main telescope drives.

The Cassegrain cable-wrap assembly is a large annulus attached to the back of the M1 Cell; of mass $1000 \mathrm{~kg}$, outer diameter $4 \mathrm{~m}$, and inner diameter $2.7 \mathrm{~m}$ to give clearance for the VIRCAM filter wheel and electronics boxes. This cable-wrap is a horseshoe-type design, allowing 1.5 revolutions of the Camera end-to-end, therefore allowing any user-selected Camera position angle on the sky followed by a 180 degree observing track. The large size of this wrap requires its own independent drive system to eliminate drag torque on the Instrument rotator axis. The Igus cable chain is supported between two frames: an outer frame is fixed to the M1 Cell, and an inner frame is mounted on a rotary bearing (separate from the instrument) driven by its own motor. The cable-chain is held captive between the two frames by a wheeled guide drum, which is dragged around by the Ubend in the cable chain, at approximately half the rate of the inner ring. The cable-wrap inner frame is electronically slaved to follow the Instrument rotator angle by an LVDT sensor mounted between the two; lanyard pull-switches stop both systems if the cable-wrap to Instrument following error exceeds $\approx 3$ degrees. Connector boxes are provided on both ends of the wrap, so if necessary the complete Cassegrain cable-wrap can be removed as a single unit (after attaching bracing bars). Normal removal of the VIRCAM leaves the cable-wrap in place.

\subsection{Telescope summary}

The overall telescope tracking and pointing performance is very good: the typical observed high-frequency tracking errors (measured from the difference between actual and demanded encoder readings) are $\approx 0.09$ arcsec rms around the Altitude and Azimuth axes, and 0.3 arcsec around Cassegrain axis; note that for conversion from axis to on-sky errors, the Azimuth error is reduced by a factor of $\cos (\mathrm{Alt})$, and the Cassegrain error is reduced by a factor of 0.03 (the field diameter in radians); thus for typical airmasses the Altitude errors contribute slightly more than Azimuth, while Cassegrain errors contribute much less. Lowfrequency errors (e.g. pointing model residuals, thermal drifts) are largely eliminated by the autoguiders.

Absolute pointing errors have been measured at $\approx 1.0$ arcsec rms in full-sky pointing runs at Altitude $\geq 25^{\circ}$. In practical operation, small non-repeatabilities in the $\mathrm{M} 1$ position from night to night can degrade this slightly, but the pointing is generally good. (The telescope normally operates with "closed-loop" pointing at each target acquisition, by applying a correction to move the guide star onto the theoretically predicted pixel on the guide chip.) Differential open-loop pointing accuracy for subdegree sized offsets is comfortably better than 1 arcsec.

The telescope offsetting performance is excellent, with slewand-settle times of $\approx 7 \mathrm{~s}$ for offsets $\sim 10$ arcmin, and about $10 \mathrm{~s}$ for degree-sized moves; the structure settles fast, with no sign of image oscillations after an offset movement.

For large-angle moves, the overheads are dominated by maximum slewing speeds, which are $2 \mathrm{deg} \mathrm{s}^{-1}$ for azimuth and altitude, and $3.6 \mathrm{deg} \mathrm{s}^{-1}$ for Cassegrain; axis accelerations are $1 \mathrm{deg} \mathrm{s}^{-2}$ for azimuth and altitude, and $1.8 \mathrm{deg} \mathrm{s}^{-2}$ for Cassegrain, thus each axis can accelerate from standstill to maximum speed in only $2 \mathrm{~s}$. These values are fast enough that the time overhead for a large-angle slew is usually dominated by the maximum speed of the azimuth axis.

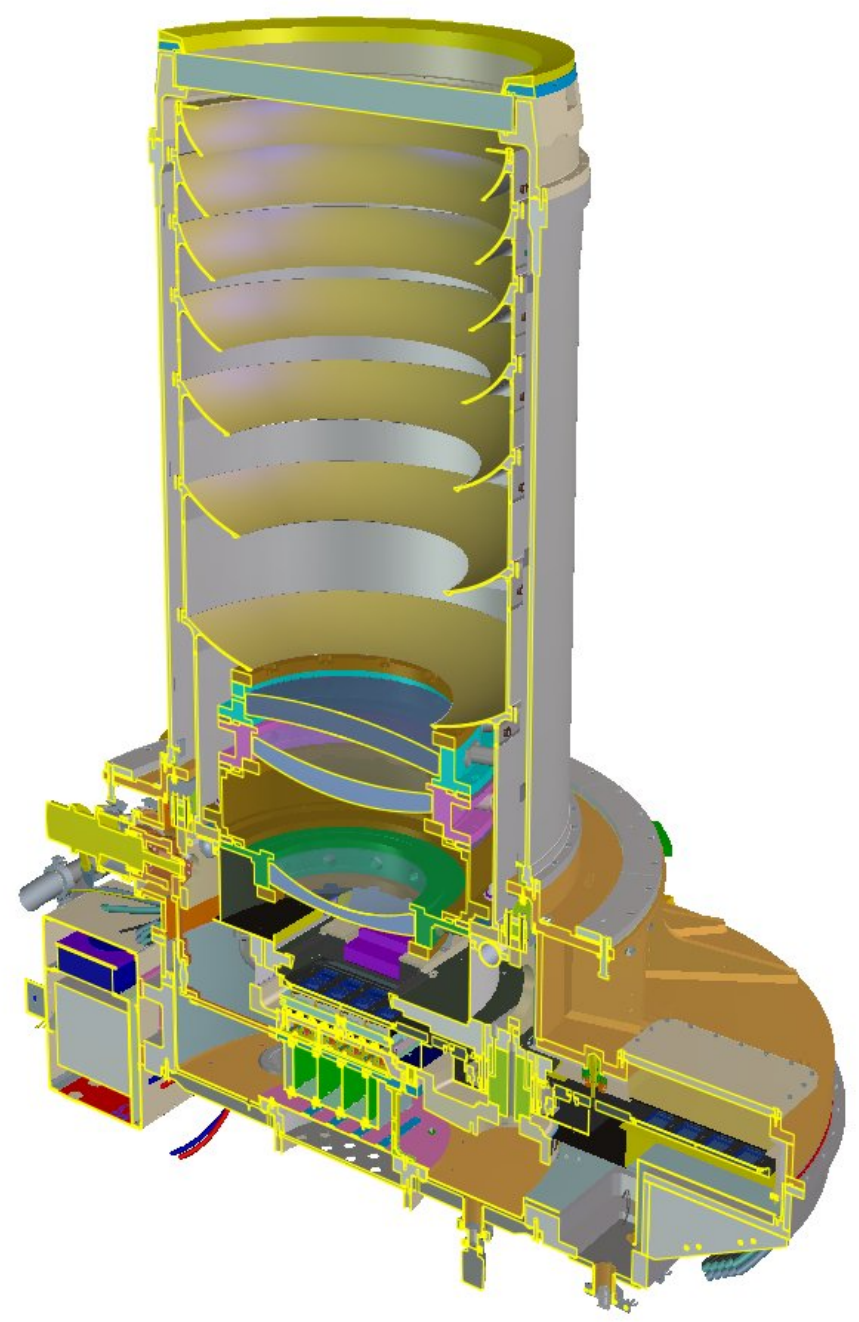

Fig. 9. Cut-away computer model of the VIRCAM (with some thermal shields and other details omitted); here the vacuum window is at the top and the filter wheel is at the bottom right. The ellipsoidal cold-baffles are seen in the upper half; lenses and window are shown in grey. A wavefront sensor unit is in purple, and some filters are seen as dark blue squares, with the detectors just below.

No significant windshake has been observed; the lowest natural frequency of the structure was modelled to be $9.2 \mathrm{~Hz}$, which is among the best for any telescope of similar aperture.

In summary, apart from occasional transient electrical glitches (e.g. defective power supply units or poor connections) affecting the control systems, the performance of the telescope structure and axes is very good. The M1 support system has also been very reliable, after fixing some infant-mortality problems in the force control electronics.

\section{IR camera}

The VISTA Infrared Camera (hereafter VIRCAM; Figs. 9, 10) is currently the world's largest and widest-field astronomical near-IR imager, with a mass of $2900 \mathrm{~kg}$, cryostat length of $2.8 \mathrm{~m}$, and a corrected field of view of 1.65 degrees diameter. The focal plane contains 16 Raytheon VIRGO HgCdTe $2048^{2}$ detectors, totalling 67 Mpixels, giving an active field of view of $0.60 \mathrm{deg}^{2}$. Further details of the design and assembly are given by Dalton et al. (2006), and commissioning results in Dalton et al. (2010).

The cryostat geometry is approximately a stepped cylinder, with diameter $1.2 \mathrm{~m}$ at the back end, and $1.1 \mathrm{~m}$ at the front; a 


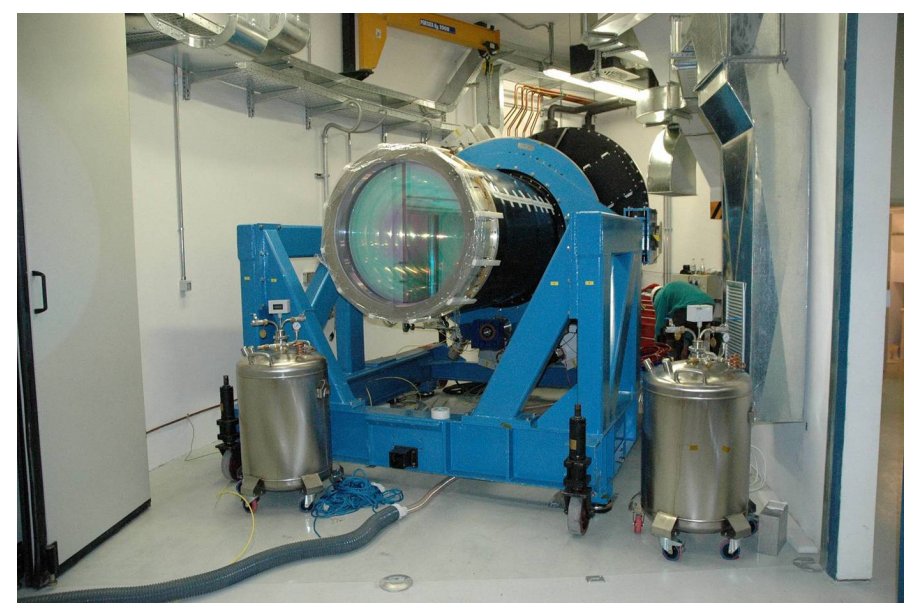

Fig. 10. VIRCAM instrument at Paranal, in its lab on the enclosure ground floor. The camera (black) is mounted on its blue handling trolley; the filter wheel bulge is visible at the back. Here a transparent protective cover is mounted over the window.

large bulge in the cryostat near the back encloses the filter wheel. The camera is mounted to the Cassegrain rotator on the back of the primary mirror cell, via a flange at approximately $80 \mathrm{~cm}$ above the cryostat back-plate.

After reflection from $\mathrm{M} 1$ and $\mathrm{M} 2$, the converging $f / 3.25$ beam enters the front of the camera via a $95 \mathrm{~cm}$ diameter Infrasil vacuum window (located approx $1 \mathrm{~m}$ above the primary mirror). The beam then passes through three Infrasil field-corrector lenses, then the filter, and finally reaches the science detectors.

Although the camera is unusually large, it is mechanically quite simple, with only one internal moving part (the filter wheel). This was a deliberate design decision, since the combination of the single-instrument telescope and the long Camera warmup/cooldown time implies that any fault requiring opening up the cryostat will lose at least 8 nights of observing time. (Notably, the autoguiders and wavefront sensors were designed with no moving parts).

\subsection{Thermal and vacuum design}

The camera internals, totalling $\approx 800 \mathrm{~kg}$ cold mass, are held at operating temperature by three Leybold 5/100T two-stage closed-cycle coolers; these are fed with Helium gas at ambient temperature and 20 bars by compressors located on the ground floor, via hoses running through all the telescope cable-wraps. The vacuum is maintained by two Leybold cryo-pumps (one operating and one redundant), using another two identical compressors: each compressor has a separate supply and return hose, thus there are ten Helium hoses in total running to/from the camera. The cryo-pumps have gate-valves so (if necessary) the getters can be warmed up and outgassed with an external pump without breaking vacuum on the main cryostat. The Helium circuits can be diverted to the Instrument lab via T-valves.

The thermal design of the camera (details in Edeson et al. 2004) is essentially a 4-layer Russian doll, as follows:

1. The outermost layer is the vacuum vessel and window. The cryostat nose (above the M1) and window edge are held at ambient temperature by low-power heater elements on the vessel, to minimise adverse local-seeing effects.

2. The second layer is a set of overlapping stainless steel radiation shields attached to the inside of the cryostat by insulating standoffs; these shields cool passively to $\approx 240 \mathrm{~K}$, and reduce parasitic heat radiating to the cold structure. (Use of multilayer insulation was considered but rejected, due to concerns about outgassing from trapped volatiles).

3. The third layer is the main cold structure operating at $\sim 100 \mathrm{~K}$ : this includes several linked Aluminium alloy support frames, to which the lens barrel, cold baffle, filter wheel and detectors are all attached. The central "optics bench" is coupled to the cooler first-stage cold heads via large copper braids. Most of the cold structure operates at 100-110 K, though there is a gradient up the cold baffle. The cold structure includes a cover which encloses all of the filter wheel; since the corrector lenses are opaque at $\lambda>3.5 \mu \mathrm{m}$, the last lens cools to $\sim 110 \mathrm{~K}$; thus the filter wheel sees only cold surfaces, and cools radiatively to approximately the same temperature as the cover.

4. The fourth and innermost thermal layer is the detector system, including the detector mounting plate and a copper "thermal plate". This thermal plate is linked by copper flexistraps to the cooler second-stage cold heads, and the detector cooling straps are connected to the thermal plate. Without heating, the thermal plate would overcool to $\sim 40 \mathrm{~K}$; a servo heater on the thermal plate controls its temperature to a setpoint of typically $68 \mathrm{~K}$ via a fast inner control loop on a Lakeshore controller. This thermal-plate setpoint is adjusted in software using a slow outer control loop to maintain the mean detector temperature at a user-defined setpoint, normally $72 \mathrm{~K}$.

Detector temperatures show quasi-static differences $\sim 1 \mathrm{~K}$ between detectors; the more important time variation is dominated by a $\sim+0.1 \mathrm{~K}$ temperature hump for a few minutes after a large rotation of the filter wheel; this occurs because out-of-beam filters sit in the cryostat bulge and are modelled to equilibrate $\sim 10 \mathrm{~K}$ warmer than the in-beam filter, so the detectors experience a slight step in radiative heating after a wheel move. The thermal control loop compensates this, but with some lag.

The cold structure is supported from the cryostat vessel via a "crown" structure of eight G10 glass-epoxy bipods; these provide good strength and low thermal conductivity. (Of the total parasitic heat into the Camera, in practice over $95 \%$ of the heat is radiated, and $<5 \%$ is conducted down the bipods.) Each bipod leg comprises 3 blades which are thin in the radial direction but very rigid in the tangential and axial directions: this allows for thermal contraction of the Aluminium cold-structure, while maintaining rigid relative positioning between the cold mass and the cryostat.

For cooldown from room temperature, it is possible to cool the camera using the closed-cycle coolers alone, but this takes approximately 7 days which is undesirably slow. To reduce cooldown time, a pre-cool pipe is permanently attached to the optical bench via conductive links; for pre-cooling, liquid nitrogen (LN2) is fed into this pipe using a semi-automated external control device, and boiled off to outdoors; this reduces the total cooldown time to around 2.5 days using approximately 400 litres of LN2.

The cold structure includes a $500 \mathrm{~W}$ warm-up heater attached to the Optics bench (fed by separate external power) which can warm the cold mass to room temperature in approximately 2 days.

Also, a dedicated battery supply is provided which feeds only the focal plane heater; in the event of a long-duration power loss which runs out the main UPS batteries, or loss of computer thermal control, this backup battery trickles approx $10 \mathrm{~W}$ of heat 
to the detectors thermal plate for up to $48 \mathrm{~h}$; this ensures that the detectors do not become the coldest point in the cryostat and become a cold-trap for outgassing contaminants.

\subsection{Vacuum window}

The VIRCAM vacuum window was probably the third most challenging component in the project, after the M1 and the 16 near-IR detectors. The window is nominally flat, made of Heraeus Infrasil IR-grade fused silica, $95 \mathrm{~cm}$ diameter and $79 \mathrm{~mm}$ thick, to provide a very conservative safety margin against the large atmospheric pressure load $(60 \mathrm{kN}$ during testing in the UK, then $45 \mathrm{kN}$ at Paranal).

The window size exceeded the maximum diameter available from Heraeus, so a smaller but thicker ingot was provided by Heraeus; this was then shipped to Corning, USA for "flowing out" into a larger thinner blank, followed by machining to final size. The blank then went to Sagem, France for polishing.

Several mishaps were encountered: the first two ingots cracked during cooling, but the third was successful. After the flowout procedure, a handling accident broke a chunk from the edge of the blank; fortunately this chunk was outside the final diameter, so this disappeared in machining to final size.

The refractive index inhomogeneities (probably due to the flow-out procedure) are non-negligible given the thick window: this was compensated in the final stage of polishing using a measured refractive index map. Sagem performed a final correction stage by first polishing the surfaces optically flat, then ion-beam polishing non-flatness onto one surface with the opposite sign to the refractive index variations.

The window is broadband anti-reflection coated, and is supported on a $91 \mathrm{~cm}$ diameter O-ring in a purpose-made Cell. Small heater elements are attached around the edge of the window, which are servo-controlled to keep the edge close to ambient temperature.

\subsection{Lens barrel}

The VIRCAM contains a 3-lens all-Infrasil field corrector; blanks were provided by Heraeus, and optically polished by Sagem, France. The lens barrel includes alignment shims, so that the final lens spacings were re-optimised after polishing of the lenses based on the measured radii of curvature (Leclerc et al. 2004).

The lenses are large, with the largest lens $582 \mathrm{~mm}$ outer diameter, therefore their cryogenic mounting required significant design attention. The lenses are supported in an Aluminium alloy barrel (Fig. 11) to match the remainder of the Camera cold structure.

From room temperature to $100 \mathrm{~K}$, the Aluminium barrel contracts by approximately $0.34 \%$ while the Infrasil lenses contract by $<0.01 \%$, so thermally compensated mounts are essential to avoid large pinch forces on the lenses when cold. Each lens is held centered in its cell by 12 radial rod+spring retainers equispaced around its circumference: the rods are made of PTFE which contracts by $1.75 \%$ over the above temperature range, relatively about 5 times the barrel. Thus, setting the rod lengths to $1 / 5$ th of the lens radius means that the linear contraction of lens+rod cancels that of the barrel, so the spring compression is almost constant with temperature. The springs are preloaded warm to provide positive centration of the lenses, and are stiff enough that the lenses decentre $\leq 70 \mu$ m under their own weight.

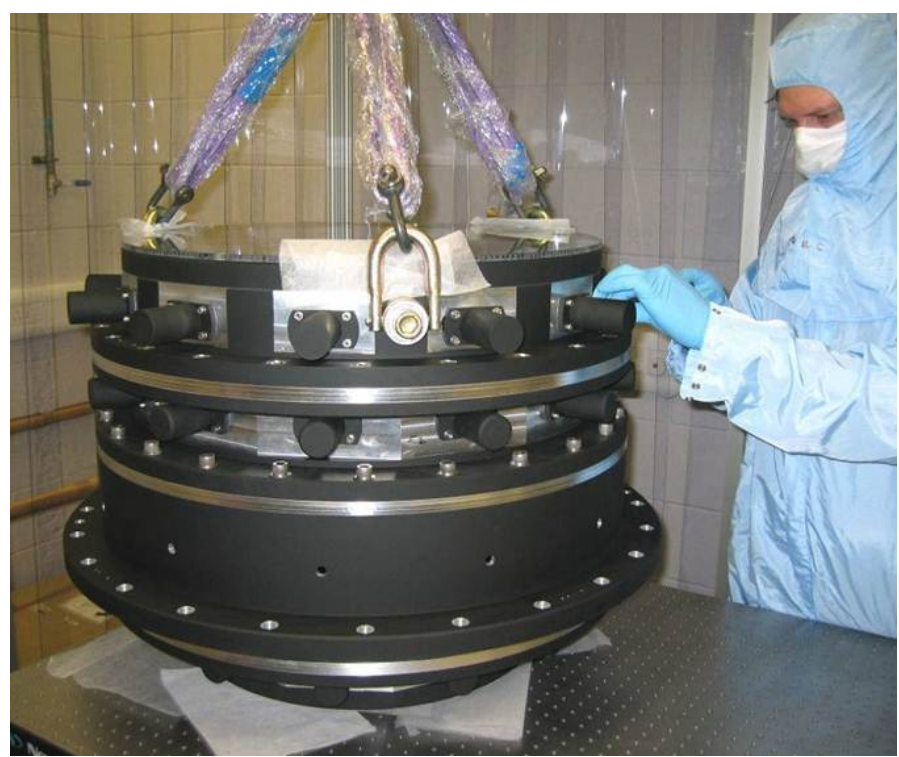

Fig. 11. VIRCAM corrector lens barrel during final assembly at UKATC, with a protective cover on top. The flange near the bottom mounts to the VIRCAM main optics bench. The cylinders extending radially out from each cell enclose the PTFE thermal compensation rods and springs.

We note that using rods alone did not ensure positive centration of the lenses, while using springs alone was not acceptable: if the springs are stiff enough to keep gravity decentre $\leq 70 \mu \mathrm{m}$, an uncompensated system would induce large compressive forces $\sim 2 \mathrm{kN}$ at each spring as the barrel contracted to operating temperature, which was shown in early modelling to be optically unacceptable. The selected combination of springs+rods in series provides modest lens stresses and good lens centration. Modelling also showed that even in an unrealistic fast-cooling scenario with the rods at $293 \mathrm{~K}$ and the lens barrel at $110 \mathrm{~K}$, the compressive forces remain well inside the safe limit for Infrasil; therefore thermal shock during camera cooldown cannot harm the lenses.

Also, each lens is thermally coupled to the barrel by copper flexi-straps which are held between the PTFE rods and the lens edge. This ensures that the lenses are thermally coupled to the barrel at 12 points, which means that the lens temperatures are nearly axisymmetric inside the optical beam; this minimises asymmetric temperature-dependent refractive index variation. (Symmetric variation is present, but is essentially pure focus shift which is corrected by active optics.)

The lens barrel is designed to minimise stray light: the lenses are generously oversized by $>20 \mathrm{~mm}$ radius beyond the optical aperture, so all lens edges are shadowed from the window or hidden from the detectors. The inward-facing surfaces of lens supports are V-grooved to minimise single-scatter stray light, and coated with Aeroglaze Z306 black paint.

\subsection{Filter wheel}

The VIRCAM filter wheel rotates to place a selected passband filter in the optical beam: it is a large annular wheel of $1.37 \mathrm{~m}$ diameter and overall mass of $75 \mathrm{~kg}$, with its rotation axis offset $500 \mathrm{~mm}$ from the optical axis. The wheel has eight main positions equispaced at 45 degrees; one is reserved for the Dark (opaque) calibration filter, thus seven sets each of 16 individual science filters are accommodated. 
The wheel is moved in open-loop using a commercial stepper motor customised for cryogenic operation. The motor drives the wheel via a Delrin polymer worm: the gear ratio is $210: 1$ and the stepper motor uses 1000 half-steps per revolution. The wheel can rotate in either direction and usually takes the shorter path, but all moves are completed by moving to a motor position +1000 counts from the target, then moving 1000 counts in the negative direction at low speed; thus all moves are terminated in a standard direction to minimise backlash errors.

A datum switch is used to initialise the zero-point of the wheel step count. To avoid a single-point failure, a second (offset) datum switch is provided, which can be selected by replugging wires outside the cryostat and telling the software that the backup datum switch is connected.

Also, a third "near-position" switch is provided which clicks on/off at every science position, 8 times per revolution. This cannot discriminate which filter is in use, and is not used in closed loop: but it provides a sanity-test against gross wheel errors, and logging the step count each time this switch changes state can provide information about wheel non-repeatability.

Each science "filter" is actually a mosaic of sixteen individual $54 \mathrm{~mm}$ square glass or Infrasil panes, one per science detector, all mounted in an Al tray using spring-loaded retainers. Currently the filter complement is $Z, Y, J, H, K_{\mathrm{s}}$, a narrowband $1.18 \mu \mathrm{m}$, and a "dual" filter with narrow-bands $0.98 \mu \mathrm{m}$ and $0.99 \mu \mathrm{m}$ covering half the detectors each. The complement of filters in the wheel can be exchanged (with the camera warm and off the telescope) via a dedicated filter access hatch on the cryostat bulge. The hatch is on the upper side of the wheel, away from the detectors, to minimise risk in case of a stray part falling from a filter tray.

The filter wheel also contains eight smaller "intermediate" slots in the V-shaped gaps between the rectangular science filters. Each intermediate slot covers only three science detectors; currently one of these slots accommodates the two high-order wavefront sensing beamsplitters (see Sect. 8), and the remaining 7 are blanked off.

The structure outside the filter wheel also includes baffling to minimise scattered light bypassing around the wheel edge and reaching the detectors. This is very successful; the measured count rate with the dark filter is $<10^{-5}$ of that through the science filters, so low that dark frames do not change if the dome lights are turned on.

\subsection{Cold baffles}

The cold baffle stack is a critical element of the Camera: it serves two functions, firstly to block unwanted heat radiation from reaching the detectors, and secondly to minimise radiative heat loss from the window into the cryostat.

The $250 \mathrm{~kg}$ cold baffle structure (Fig. 12) is made of Al alloy: the geometry is an outer support cylinder, with seven nested ellipsoidal baffles inside the cylinder, and one flat annulus on top. The flat annulus with aperture diameter $812 \mathrm{~mm}$ defines the entrance beam to the camera; the ellipsoids are sized so that they clear any ray from the top baffle to the detectors.

The baffle support cylinder is attached at the bottom end to the main Optics bench: due to the substantial overall height $1.82 \mathrm{~m}$, it has a significant temperature gradient, running from $100 \mathrm{~K}$ at the bottom to $160 \mathrm{~K}$ at the top, but the top end by design remains cold enough that its own thermal emission contributes negligibly to the detector background. Keeping the baffle top acceptably cold required a substantial wall thickness for the baffle support cylinder, hence the substantial baffle mass.

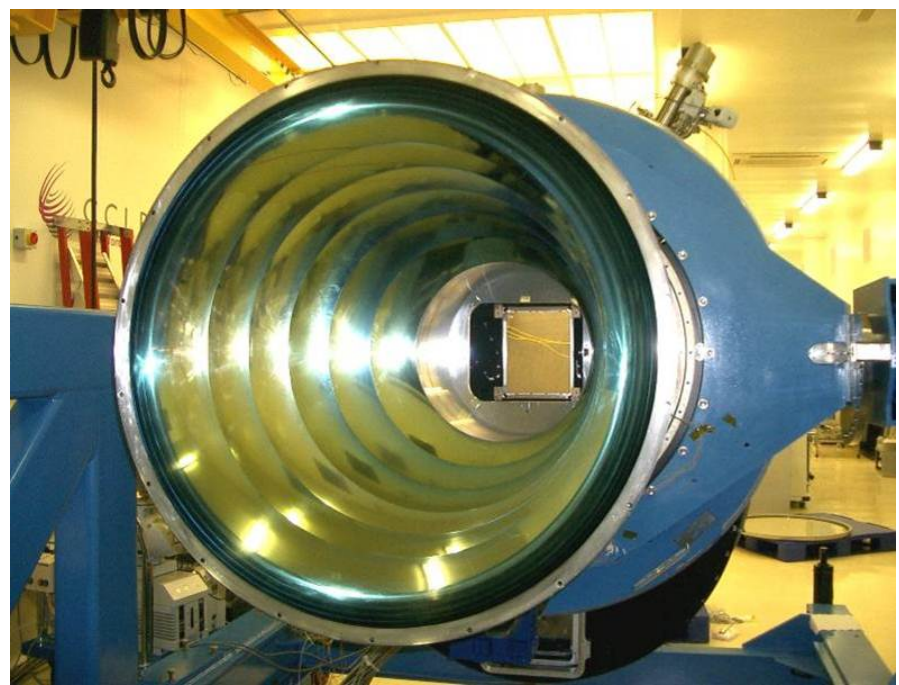

Fig. 12. VIRCAM cold baffle, with the cryostat nose and window not present.

A significant design challenge was to avoid overcooling of the window: the Infrasil window is opaque at thermal-IR wavelengths $>4 \mu \mathrm{m}$, and therefore emits $\sim 100 \mathrm{~W}$ of blackbody radiation downward into the cryostat. If most of this were absorbed, the centre of the window would chill $>10^{\circ} \mathrm{C}$ below ambient temperature, leading to a severe problem with dewing or frosting on the window's outer surface. Since Infrasil is a poor heat conductor, heating the window edge would not remedy this. Therefore, most of the heat energy radiated by the window must be reflected back to the window.

The ellipsoids in the baffle are shaped so as to reflect as much heat as possible back to the window. However, simple metallic heat reflectors would not be acceptable at $K_{\mathrm{s}}$ band, since emission from the dome can reach the detectors via two reflections in the baffles and M2. As viewed from the detectors, the cold baffles reflected in M2 subtend around $15 \%$ of the total M2 solid angle, so fully-reflective baffles would give almost $15 \%$ additive emissivity contribution, clearly unacceptable.

The solution to these two opposing requirements uses the fact that for a room-temperature blackbody, almost all the radiated power is emitted at long wavelengths $\lambda>5 \mu \mathrm{m}$, much longer than the science bands: therefore, the adopted solution is to apply a dichroic coating on the baffles which is a good reflector at $\lambda>4 \mu \mathrm{m}$ but is strongly absorbing at science wavelengths $<2.5 \mu \mathrm{m}$. This custom coating was developed specially for VIRCAM and applied to the upper surfaces of the ellipsoid baffles by Reynard Corp. of California.

All surfaces visible from the detectors, i.e. the inner face of the cylinder, and the downward-facing sides of the ellipsoid baffles, are finished with Aeroglaze Z306 black paint for maximum absorption of scattered light: also, the ellipsoid baffles are spaced so that no point on the window can see the inner face of the cylinder, therefore the only single-scatter paths from the window to the detectors are the sharp-edge tips of the ellipsoid baffles.

\subsection{Detectors}

The camera's "retina" comprises sixteen Raytheon VIRGO $\mathrm{HgCdTe}$ infrared detectors (Love et al. 2004), each of $2048^{2}$ format and $20 \mu \mathrm{m}$ pixel size. For efficient tiling of sky, the detectors are arranged in a $4 \times 4$ rectangular grid, with spacing of $0.9 \times$ the 
active width in the detectors $y$-direction (which is cryostat $-x$ ) and $0.425 \times$ active width in the orthogonal direction.

The detectors have an operating wavelength range (halfpeak) $\approx 0.75-2.45 \mu \mathrm{m}$, and the quantum efficiency is very high from 1.0 to $2.35 \mu \mathrm{m}$; measured QE is near $90 \%$ for most of the detectors across this range (Bezawada et al. 2004). At the short end there is a gradual QE rolloff to $\sim 75 \%$ at $0.8 \mu \mathrm{m}$, and a sharp cutoff below $0.75 \mu \mathrm{m}$.

The total of 67 Mpixels makes this the largest near-IR focal plane in astronomical use; a number of other astronomical instruments have four $2048^{2}$ detectors giving 16.8 Mpix, but VIRCAM is the only instrument to exceed 17 Mpix at the present time.

The detectors comprise a CdZnTe "substrate" above the active layer, then the active $\mathrm{HgCdTe}$ layer; this is bump-bonded via 4 million individual Indium bumps to the silicon multiplexer which reads out the signals. The silicon is attached via epoxy glue to a thick Molybdenum back-plate: the Mo is a very good CTE match to CdZnTe, and thus forces the silicon to contract at the same rate as the rest of the detector during cooldown.

Readout noise is typically $24 e^{-}$rms for a single integration with double-correlated sampling (well below sky noise for broadband filters); dark current is typically $0.2 e^{-} \mathrm{s}^{-1}$, with a tail to larger values. The detectors do have a significant number of cosmetic defects, with typically 1 percent dead or hot pixels; one detector has some large "dead patches" totalling $\sim 3$ percent of its area. Most of the dead or hot pixels are time-invariant, so the effect largely disappears in post-processing of jittered frames; however, this does mean that it is advisable to observe at least 4 distinct jitter positions.

The 16 individual science detectors (Fig. 13) are bolted to a thick Molybdenum mounting plate (matching the detector backplates) which has a waffle-type lightweighting pattern on its back side, and includes feedthrough slots for the detector ribbon cables. The plate was machined accurately flat, and detector thicknesses matched so all pixels are coplanar within $\pm 12.5 \mu \mathrm{m}$. The plate is held in position from the Al support frame via a central boss providing centration, and three titanium "knife blades" at the edge constraining rotation and tip/tilt. The mounting arrangement allows for differential contraction between the Mo plate and the $\mathrm{Al}$ support structure with minimal stress on the Mo plate.

A cold electronics box attaches to the back of the Al detector support frame. If necessary, the complete subsystem of detectors, Mo plate, support frame and electronics box can be removed via the back of the Camera as a single $54 \mathrm{~kg}$ assembly, after warming up the Camera and removing the cryostat backplate and thermal shields.

\subsection{Detector electronics and readout}

The detectors are controlled and read out by ESO IRACE (InfraRed Array Control Electronics) controllers. One master box is used for clock signals, driving four slave controllers each controlling a column of four detectors. Each detector has sixteen parallel readout channels, each 128 pixels wide by 2048 pixels high, thus overall there are 256 parallel readout channels.

The default readout time is $1 \mathrm{~s}$ (pixel rate $294 \mathrm{kHz}$ per channel, including reference pixels). The standard readout mode is double-correlated sampling; for a given detector integration time (DIT), the detectors are reset, then a non-destructive read is immediately done, followed by a wait for DIT $-1 \mathrm{~s}$ and another read. (Windowed readout is possible for faster operation, but is

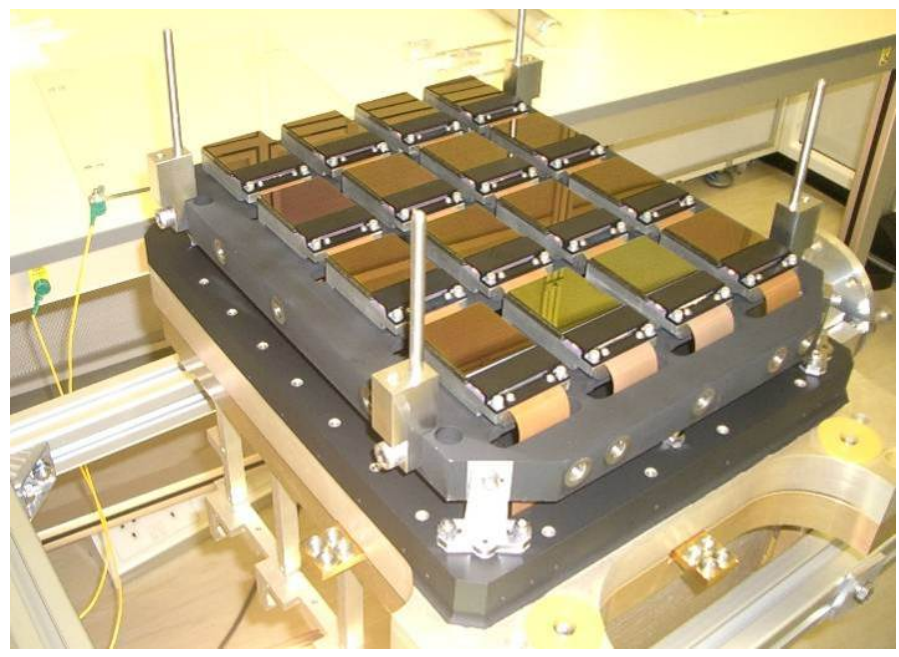

Fig. 13. VIRCAM focal plane. The active areas of the 16 detectors are seen with the bronze-coloured anti-reflection coating. Black covers are over the detector electronics, and the ribbon cables are seen leading to cold electronics below. One of the three knife-blade supports can be seen at the front corner. The four vertical rods are temporary guides for a protective cover.

not offered as a standard operating mode; this is constrained to read the same sub-window on all 16 detectors.)

The median system gain is $4.19 e^{-} / \mathrm{ADU}$, and readout noise is typically 24 electrons for a single integration with doublecorrelated sampling; this is well below the sky noise for typical broadband exposures.

Detector linearity and dark pixel calibration is done using the flat-field screen and calibration lamps inside the enclosure (see Sect. 12). Non-linearity is significant, typically 2-4 percent at 10000 ADU flux level with one detector at 10 percent, but is fairly stable over time and within each detector. The nonlinearity is corrected in the data processing pipeline using polynomial fits to the observed linearity sequences.

Normal exposures are done by co-adding several individual detector integrations in the IRACE electronics before saving to disk (e.g. $6 \times 10 \mathrm{~s}$ for $K_{\mathrm{s}}$ band, or $3 \times 20 \mathrm{~s}$ for $J$ ). This reduces data volume and overheads, since consecutive integrations coadded in electronics have an overhead of $2 \mathrm{~s}$ each, while the overhead in the IRACE for processing a complete exposure and clearing buffers is $4 \mathrm{~s}$; this sets the minimum time between consecutive exposures, since the $\mathrm{N}+1$ th exposure cannot start until the $N$ th exposure has been cleared. However, telescope jitter/offset moves are operated in parallel with the clearing of IRACE buffers, and these usually take longer than the $4 \mathrm{~s}$, so this rarely adds overhead.

\subsection{Autoguider and wavefront sensors}

The VIRCAM contains six auxiliary CCDs (two units, each with two WFS CCDs and one autoguider CCD) to provide autoguiding and wavefront sensing; however, since these are functionally part of the telescope active optics system rather than the Camera, we postpone description of these until the next Sect. 8 .

\subsection{Camera optical testing}

The Camera was challenging to test in the lab, due to the long cooldown/warmup time, and the fact that the Camera and Telescope form a linked optical system: thus, for image quality testing we constructed a "telescope simulator" which provided 
input beams with deliberate aberrations closely approximating those of the real telescope at two specific field locations, "nearaxis" at $30 \mathrm{~mm}$ and "off-axis" at $112 \mathrm{~mm}$ (Dalton et al. 2010).

The telescope simulator comprised a small Cassegrain-like telescope with $0.6 \mathrm{~m}$ spherical primary mirror and $0.2 \mathrm{~m}$ spherical secondary, together with small corrector lenses. The corrector lenses included a cylinder lens to generate the required large off-axis astigmatism; by suitable re-spacing of these lenses, this simulator with a pinhole source generated an $f / 3.25$ converging beam into the Camera, with aberrations close to the real VISTA $\mathrm{M} 1 / \mathrm{M} 2$ at either 0.14 or 0.53 degrees off-axis.

For these tests, the Camera was built in a "short nose" configuration with the real lens barrel but without the cold baffle and using a smaller-diameter $60 \mathrm{~cm} \mathrm{BK7}$ test window, since the real Infrasil window (as expected) was not delivered until the final stages of Camera testing. All tests were done at $J$-band only for simplicity.

This camera optical testing procedure consumed a significant amount of time and effort, and due to the challenging tolerances for relative alignments of the test source, it did not quite meet the target accuracy level we were initially hoping for: however, it did achieve the key purpose of giving an end-to-end independent proof that there was no severe error in the camera optics, in advance of flying the camera to Chile.

During commissioning on-sky, we found a moderate astigmatic term $\sim 300 \mathrm{~nm}$ which co-rotates with the Camera and is roughly constant across the field of view; the cause is not known for certain, but is suspected to relate to a thermal gradient across lens L3 and consequent refractive index gradient. This was corrected quasi-automatically by the active optics system, but to eliminate correction delay after a slew we added an active force term to the M1 in software which co-rotates with the Camera.

\subsection{Handling and transport}

A large purpose-built earthquake-resistant camera trolley was made, and this is used both for camera assembly at RAL Space and off-telescope maintenance at Paranal. The trolley includes pitch and roll bearings to re-orient the camera during assembly and maintenance operations.

For transport, it was decided to fly the camera to Chile fully assembled including detectors. A customised container was designed which takes the camera on the trolley. The complete package of Camera, trolley and container weighed over 9 tonnes, and was designed to (just) fit through the side door of a Boeing 747 cargo aircraft. For safety, the trolley was linked to the container floor via substantial wire-rope shock isolators; these were specified so that a computer-modelled $20 \mathrm{~cm}$ free-fall accident should inflict no more than $5 \mathrm{~g}$ peak deceleration on the Camera. In reality, the journey was smooth and the camera shock recorders measured no shock exceeding $0.5 \mathrm{~g}$ during its flight to Santiago and subsequent road transport to Paranal.

Due to the long camera nose, mounting the Camera onto the telescope is necessarily done with the telescope pinned horizonpointing, using the main enclosure crane. Since the camera centre of gravity is inside the Cassegrain rotator, this uses a purposemade lifting arm which attaches around the back face of the cryostat and has the lifting eye $3 \mathrm{~m}$ above the combined centre of gravity, above the M1 Cell.

\subsection{Camera shim}

For adjustment of Camera position, the design includes a substantial shim (made of nine sectors) between the instrument flange and the Cassegrain rotator; this had a nominal thickness of
$25 \mathrm{~mm}$ with a possible range from $5-45 \mathrm{~mm}$. The purpose was twofold: firstly, the wide range allowed potential spherical aberration compensation in the event of conic constant error in either mirror, by a combination of axial shift of the whole VIRCAM and refocussing $\mathrm{M} 2$. In reality, the as-built spherical aberration of $\mathrm{M} 1+\mathrm{M} 2$ was very close to predictions; however the as-built VIRCAM window was $1.5 \mathrm{~mm}$ thinner than designed due to extra fine-polishing to remove surface defects, so the baseline $25 \mathrm{~mm}$ shim thickness was reduced by $0.5 \mathrm{~mm}$ to compensate the thinner window. Secondly, changing to a new shim with a slight wedge angle allowed correction of the detector plane orthogonal to the Cassegrain axis, avoiding the risky operation of opening the cryostat and adjusting detector mounts. This tilt adjustment was used: near the end of commissioning, analysis of science images indicated a small residual focal tilt (see Sect. 8), so a second shim was made with the same mean thickness but a $150 \mu \mathrm{rad}$ wedge angle; this was installed in early 2010 and reduced focal tilt to a negligible value.

\subsection{Camera performance}

Apart from several minor teething problems during commissioning, mainly regarding electronics problems and inadequate initial glycol supply, the VIRCAM has generally been very reliable since the end of the commissioning period.

The cold baffle has proved to be a successful design: the observed $K_{\mathrm{s}}$-band background is in line with the computer modelling, while the window centre is estimated to be $\sim 4{ }^{\circ} \mathrm{C}$ below ambient temperature. Dewing of the window has occurred a few times to date during rapid increases in humidity, but the window has been successfully cleaned by careful hand-washing without degradation of the coating.

The vacuum and thermal design has proved successful, and the Camera can remain cold for the 1-year intervals between cryocooler major overhauls. There have been no significant outgassing or contamination problems, and since the Camera departed from the UK there has never been any re-alignment required of lenses or detectors inside the Camera, and only one internal intervention to fix a problem with the filter wheel. Sensitivity and image quality are described in Sect. 13.

\section{Active optics}

VISTA has a fairly sophisticated guiding and active optics system, which includes a total of six auxiliary CCD detectors (inside the VIRCAM) totalling 24 Mpixels. The purpose is to control the telescope guiding, and the position of the secondary mirror (in 5 axes) and the figure of the primary mirror (in up to 18 eigenmodes) to maintain near-optimal image quality.

As a notable feature, VISTA is probably the first telescope ever to deliver closed-loop 5-axis collimation ${ }^{3}$ of the secondary mirror in parallel with observing; traditional telescopes only used an occasional focus shift, while most modern telescopes control 3 degrees of freedom of M2 in closed loop: usually, focus and $x / y$ rotations around M2 centre of curvature ${ }^{4}$.

\footnotetext{
3 Of the 6 rigid-body degrees of freedom, small rotations around the $z$-axis are irrelevant, so 5-axis control is required for complete collimation.

4 We note that the VLT does occasional on-sky 5-axis collimation; however sensing the 4th and 5th degrees of freedom (M2 tilt around coma neutral point) requires stepping the single guide/WFS probe to multiple locations around the field edge, so the VLT cannot do closedloop M2 tilt control during science observing. Due to the $f / 15$ system and narrower field, the VLT is much less sensitive than VISTA to misalignment in these axes.
} 
The VISTA primary mirror is only moderately thin by modern standards: its diameter/thickness ratio is 24, compared to values of 48 for the VLTs, 18 for the NTT and 6-10 for pre-1980 $4 \mathrm{~m}$ class telescopes. This is beneficial, since the M1 is flexible enough that the figure can be modified with moderate active forces, but not so flexible that very high accuracy is required for the support forces.

However, the very fast $\mathrm{f} / 1$ primary mirror and fairly fast $\mathrm{f} / 3.25$ system focal ratio mean that the system is extremely sensitive to misalignment and despacing between the M1 and M2: the image quality budget requires that the M1-M2 spacing is correct to $\approx 3 \mu \mathrm{m}$ (i.e. fractional accuracy 1 part per million), centration to $\approx 20 \mu \mathrm{m}$, and tilt to a few arcsec. It was felt unrealistic to maintain these numbers with purely open-loop control, therefore active measurement and control of the M2 position is mandatory. The IR Camera includes a number of wavefront sensors and autoguider units to provide the required corrections.

We show in Sect. 8.1 below that measurement of M2 collimation requires measurement of only the low-order optical aberrations (defocus, 3rd order coma and 3rd order astigmatism), but these must be measured at two locations widely spaced in the field, in order to disentangle the effect of M2 tilt around the coma neutral point.

Measuring the figure of the M1 is best done by a single sensor near-axis, but up to 18 bending modes are required; since the altitude dependence is relatively repeatable, this can run mainly with lookup-table control, using only occasional measurements, mainly to eliminate any long-term drift in the M1 actuator load cells.

Due to these differences in cadence and mode requirements, the wavefront sensing functions were split between separate loworder and high-order wavefront sensors. In detail, there are two autoguiders (AG), two low-order wavefront sensors (LOWFS), and two high-order wavefront sensors (HOWFS) units; the autoguiders and LOWFS are joined in two LOWFS+AG units with a common housing. The low-order wavefront sensors are required to run quasi-continuously (around 1 cycle per minute) to provide closed-loop focus and collimation of M2; while the high-order sensor(s) run only "on demand", typically once every evening twilight and many times on occasional engineering nights; the latter data are then fitted off-line to build the lookup table for M1 active forces.

\subsection{The 5-axis collimation and sensor locations}

It is well known that for a 2-mirror telescope, despace of the secondary mirror produces defocus, and decentre of the secondary mirror produces mainly 3 rd order coma $\left(Z_{7}\right.$ and $Z_{8}$ in our Zernike polynomial convention); the coma difference from an ideal system is nearly independent of field angle, so a wavefront sensor anywhere in the field is sufficient. Most modern 2-mirror telescopes correct the latter by rotating their secondary mirror around its centre of curvature, which gives almost pure coma correction and negligible image shift.

However, defocus and coma information can only correct 3 of the $5 \mathrm{M} 2$ degrees of freedom: applying this correction leaves the M1 and M2 axes crossing at the coma neutral point which is typically close to the primary focus (for VISTA the coma neutral point is $1.05 \mathrm{~m}$ above the M2 pole), but leaves tilt of M2 around the coma neutral point unconstrained (McLeod 1996; Noethe \& Guisard 2000). These authors showed that rotation of the M2 around the coma neutral point adds 3rd order astigmatism which is bi-linear in M2 tilt and field angle (therefore has negligible effect on-axis). We ran a Zemax analysis to verify that essentially the same behaviour is found for the VISTA system including the VIRCAM field corrector, and the aberration differences (relative to a perfectly aligned system) are nearly achromatic. Thus, offaxis sensor(s) are required for full collimation, but the choice of wavelength is relatively unconstrained. In principle it is possible to measure M2 tilt with only one off-axis sensor: however, with one sensor the M2 tilt is degenerate with astigmatic figure error on the mirror(s), which produces an astigmatic term nearly constant across the field of view; correcting a real M2 tilt with M1 forces, or vice versa, would double the astigmatism at a field point diametrically opposite to the wavefront sensor, clearly a very undesirable result.

Thus, we decided to use two low-order wavefront sensors in VIRCAM, located at diametrically opposite points near the edge of the field of view; the LOWFS hardware is described below, along with the autoguiders which are co-mounted.

\subsection{Low-order wavefront sensors and autoguiders}

The purpose of the low-order wavefront sensors (LOWFS) is to deliver measurements every few minutes to maintain correct position of the M2 in all 5 degrees of freedom, while the autoguiders (AG) compensate for slow tracking drift e.g. flexure or thermal residuals from the open-loop pointing model. These must operate in parallel with science observing, but the sensors must reside behind the corrector lenses inside the cryostat, so no moving probes were permitted. Thus, the LOWFSs and AGs require a wide field of view, sufficient to provide $>99 \%$ probability of finding a usable star even at the Galactic poles, assuming a $45 \mathrm{~s}$ exposure for the LOWFSs and $0.2 \mathrm{~s}$ for the AG.

This is achieved by picking off patches of sky which are inside the circular corrector field but outside the rectangular science field, and using CCDs operating at far-red wavelengths for acceptable image quality and reduced sensitivity to moonlight. (Near IR sensors were ruled out for both cost and sky background reasons.) All CCDs are E2V 42-40 2048 ${ }^{2}$ deepdepletion devices for optimal red performance; there are six CCDs, one for each AG and two for each LOWFS.

Physically, one LOWFS and one AG are combined into a common housing with three CCDs to form a "LOWFS-AG unit" (mass $2.2 \mathrm{~kg}$ ), and two identical LOWFS-AG units are used; each LOWFS-AG unit includes a housing containing a pickoff mirror, filter, beamsplitter cuboid, one autoguider CCD and two LOWFS CCDs, and a heater element to warm the CCDs to $\sim 150 \mathrm{~K}$. More details of the hardware are given by Clark et al. (2004).

The two LOWFS-AG units are mounted 180 degrees apart on an aluminium plate (with a hole for the science beam) just above the filter wheel. The VIRCAM cold structure is designed so that the LOWFS-AG mounting plate is bolted to the IR detector support frame via three thick pillars in a near-equilateral triangle; one pillar goes through the hole in the centre of the filter wheel, while the other two are outside the wheel. This geometry provides negligible differential flexure between the LOWFS-AG units and the science detectors, as required.

After the pickoff mirror, the beam into the LOWFS-AG unit passes through a fixed filter (bandpass 720-920 nm), then through a cuboid fused-silica beamsplitter (these elements are common to the LOWFS and AG detectors). The beamsplitter coating changes along the long axis of the cuboid: one end divides the light equally between the pair of LOWFS CCDs, while the other end is fully reflective and feeds the autoguider CCD (the extra reflection just helps packaging). The two LOWFS detectors are mounted $\pm 1 \mathrm{~mm}$ respectively before/after the nominal 
best focus location, so all stars image into small doughnuts defocused to a diameter of $0.3 \mathrm{~mm}$ or 5 arcsec. (In principle measuring only one side of focus is sufficient, e.g. Tokovinin \& Heathcote 2006, but our implementation measuring both sides of focus is generally more robust and provides reduced crosstalk if multiple aberrations are present.) The autoguider CCD is physically identical to the LOWFS CCDs, but operated in frametransfer mode with half the area used as storage to increase the duty cycle; maximum rate is around 5 frames/s, though usually 1-2 frames/s is selected.

With a pixel size of $13.5 \mu \mathrm{m}$ or 0.229 arcsec, each LOWFS unit views a square $7.8 \times 7.8$ arcmin of sky; this is sufficient to almost guarantee finding a usable star $(I \lesssim 15.5)$ at any telescope pointing, even near a Galactic pole. The LOWFS (and AG) detectors have no shutter, but the LOWFS readout is windowed down to typically a $100 \times 100$ box around the selected star; this reads out in $\ll 1 \mathrm{~s}$, fast enough that image trailing is barely detectable.

Normally only one autoguider is operational, whichever one has a brighter star; the use of two autoguiders gives identical LOWFS/AG units, wider sky coverage and partial redundancy against one AG failure. Both AGs can be run simultaneously in engineering mode. (In principle, two AGs could give closed-loop control of the Cassegrain rotator; however this is unnecessary given the very good open-loop performance of the Cassegrain rotator and rather short single VIRCAM exposures, and the control software does not allow this at present.)

Care was taken to minimise adverse electromagnetic interference and ghosting effects from the LOWFS/AG units to the science detectors: their placement above the filter wheel is well shielded from the science detectors, and the housing near the science beam has a sawtooth face to minimise scattered light. The LOWFS/AG wiring exits the cryostat radially, while the science detector wiring exits axially at the back-end to maximise separation. Each LOWFS/AG filter comprises $3 \mathrm{~mm}$ of Schott RG9 glass (transmitting 720-1050 nm) with a short-pass $920 \mathrm{~nm}$ coating on the second surface to reduce sky background. The RG9 is strongly absorbing at $\lambda>1.1 \mu \mathrm{m}$ so $J, H, K_{\mathrm{s}}$ bands are reflected by the coating but absorbed by a double-pass through the glass; $Z$-band of course is absorbed by the CCDs. The LOWFS filter thus behaves as a retro-reflector around $Y$ band, but this has not shown significant ghost problems so far.

\subsection{High-order wavefront sensors}

The high-order wavefront sensing only runs "on demand" by repointing the telescope at a suitable bright star. For simplicity, a novel beam-splitter cuboid was designed which fits into unused space in the filter wheel (in one of the V-shaped gaps between science filters), and produces a pair of offset above/below focus images on a science detector.

The beam-splitter comprises a cemented cuboid with two tilted plano part-reflective surfaces immersed inside it. The overall thickness is $\approx 6 \mathrm{~mm}$ thicker than a science filter; this extra silica produces a focus shift so the straight-through image has a best-focus about $2 \mathrm{~mm}$ below the science detector. The spacing of the part-reflective surfaces is chosen so the doubly-reflected path produces an image which has best-focus $2 \mathrm{~mm}$ above the science detector, and is also offset laterally by 100 pixels. The upper face of the cuboid has a regular $J$-band coating.

Thus, to operate the high-order wavefront sensor, the filter wheel is rotated to centre the cuboid above a selected pixel on the science array, and the telescope is re-pointed to put this star close to the selected pixel; then a $60 \mathrm{~s}$ exposure is taken to average out atmospheric variations. This produces a pair of offset doughnut images on the science detector, which are then analysed (see below) to measure the wavefront aberrations.

In practice, we supplied two identical cuboids which are mounted at different radii from the axis of the filter wheel; as the wheel rotates, each of these travels an arc crossing four science detectors, so by a suitable choice of wheel angle and telescope pointing, any one of eight defined spots on different science detectors can be used for high-order sensing. Normally a default spot near-axis is used, but all eight accessible spots were used occasionally during commissioning.

This high-order WFS is a simple and elegant solution which uses no additional moving parts, no additional detectors, while delivering good coverage of field positions. No motion of the secondary mirror is required - the observed pre/post focus offsets are determined purely by the dimensions of the beamsplitter cuboid, so as long as the cuboid is manufactured correctly it does not require precise location in any direction.

\subsection{Image analysis}

Both wavefront sensors use a common image-analysis package to analyse a pair of above/below focus doughnut images and estimate wavefront coefficients in various modes. In summary, a subroutine is provided which given any vector of Zernike coefficients: simulates a pair of doughnut images using the known defocus distances and system $f$-ratio, using geometrical optics ray-shooting and Gaussian blurring for seeing; then, a NelderMead simplex algorithm searches the many-dimensional space of Zernike coefficients for a minimum $\chi^{2}$ between observed and modelled images.

This "forward" search algorithm is relatively slow compared to "backwards" methods which attempt to reconstruct a wavefront directly from the data, but it is robust against fairly bad aberrations, and generally converges in $\sim 15 \mathrm{~s}$ for the low-order WFS and $\sim 500 \mathrm{~s}$ for the high-order WFS (with many more modes). These time delays are acceptable since the low-order WFS runs in parallel with observing, while the high-order WFS usually runs in bright twilight before science observing starts.

\subsection{Applied corrections}

The degrees of freedom controlled by the active optics system are the position of M2 in five axes, and the shape of M1 in 17 degrees of freedom (counting non-axisymmetric modes as two, with independent $\cos m \phi$ and $\sin m \phi$ components). Here the mode $B_{m, 1}$ is defined to be the softest mirror mode of azimuthal symmetry $m$; that is, the force pattern of the form $F_{i} \cos (m \phi)$ where $F_{i}$ for $(i=1 \ldots 4)$ are arbitrary forces per ring; which produces the largest ratio of rms deflection per unit rms force. The $B_{m, 2}$ mode is the next softest, orthogonal to $B_{m, 1}$. In order of increasing stiffness, the M1 bending modes used are as follows:

1. $B_{2,1}$ is the M1 lowest-order mode of symmetry $m=2$ (very similar to Zernike astigmatism).

2. $B_{3,1}$ is the lowest-order M1 $m=3$ trefoil-like bending mode.

3. $B_{4,1}$ is the lowest-order M1 $m=4$ quatrefoil-like mode.

4. $B_{2,2}$ is the M1 next-to-leading order mode of azimuthal symmetry 2 .

5. $B_{5,1}$ is the lowest order $m=5$ pentafoil-like mode.

6. $B_{6,1}$ is the lowest order hexafoil mode.

7. $B_{3,2}$ is the next-to-leading order $m=3$ bending mode.

8. $Z_{11}$ is Zernike spherical aberration.

9. $B_{1,2}$ is an M1 next-to-leading order coma-like mode. 
Two other possible M1 modes are not used: the $B_{0,1}$ mode is very similar to Zernike defocus, and the $B_{1,1}$ mode is very similar to Zernike coma. These modes are not applied as M1 forces, but are corrected by the M2 only, to avoid a possible runaway situation with opposing M1 and M2 corrections. All the above modes and matching force patterns were derived from finite-element modelling of the primary mirror using the pre-defined positions of the axial supports.

In normal operation, the five M2 axes and just two M1 modes (the pair of $B_{2,1}$ astigmatic modes) are controlled in closed-loop, while all higher M1 modes are controlled in open-loop via the lookup table, including a once-nightly additive correction from the most recent high order wavefront sensor measurement.

The mapping from wavefront sensor measurements to applied corrections is slightly complicated, and is essentially applied in three "layers". For operator convenience, all tables and corrections are stored and displayed as delta-wavefront per mode in nanometres rms, and then converted into M2 position offsets or M1 force patterns when sent to the hardware.

The first layer comprises a lookup table, actually a set of polynomial fit coefficients, one for every mode above. In principle the software can handle up to 4th order Legendre polynomials in Altitude and a linear term in temperature; but in practice a constant + linear Altitude dependence is a good enough fit for most modes, with quadratic terms only for defocus and one other.

The second layer is another additive constant per mode, measured from the high-order wavefront sensor. The third layer is the closed-loop cumulative correction derived from the low-order wavefront sensors. The sum of all three layers is actually sent to the system, but storing the layers separately has two advantages: firstly the lookup table updates immediately after a slew (keeping the previous additive LOWFS correction), so for moderate altitude changes $\lesssim 15 \mathrm{deg}$, observing can resume immediately without waiting for a new LOWFS correction; secondly, this setup ensures a simple un-do operation in the event of a bad wavefront measurement.

For the closed-loop measurements, the average of the two low-order wavefront sensors is used to give focus $Z_{4}$ and coma $Z_{7}, Z_{8}$ corrections to $\mathrm{M} 2$ position, and the two $B_{2,1} \mathrm{M} 1$ force corrections. The difference of the two astigmatism measurements between the two wavefront sensors (suitably rotated) is used to derive M2 tilt correction (rotation around the coma neutral point).

\subsection{M1/camera collimation and focus gradients}

The closed-loop procedure above is necessary and sufficient to align the M2 to a position which gives the as-predicted coma and astigmatism for any given M1 and Camera position: if the M1 and Camera are almost co-axial, this is sufficient to collimate M2 to the same axis and the collimation is essentially ideal.

However, if the M1 and Camera axes are misaligned to each other, there is generally no M2 position which can eliminate focus gradient and field-dependent astigmatism at the same time: centring M2 around its centre of curvature will still eliminate excess coma, but to correct either focus gradient or field-dependent astigmatism one must then rotate $\mathrm{M} 2$ around the coma neutral point: and the required M2 tilt vectors are different so one can eliminate either astigmatism or focus gradient, but not both.

The procedure is also complicated for an Altazimuth system: since the Camera rotates about the $z$-axis but M1 does not, it can be seen that both the M1 and Camera must be separately aligned to the Cassegrain rotator axis to deliver a well aligned system at any sky position; and it is the M1 hyperboloid axis which is relevant, not the centre of the hole, so this can only be determined by on-sky observations.

Our LOWFS system is set up to move M2 to deliver astigmatism matching its ideal well-aligned value (not zero), because measuring a general focus gradient in one shot requires 3 wavefront sensors, not 2; thus, if the M1 and Camera are not well aligned, a residual focus gradient will remain after active optics correction of the M2 position; this is near zero on-axis due to the symmetry of the LOWFSs. This focus gradient will decompose into two distinct $x, y$ vectors: one vector fixed to the M1 Cell (caused by fixed misalignment between the M1 axis and the rotator axis), and another vector co-rotating with the Camera (caused by misalignment between the Camera axis and the rotator axis). Since the LOWFSs also co-rotate with the Camera, the M1 misalignment term is observed as a focus difference between the two LOWFSs with sinusoidal dependence on rotator angle. The misaligment term co-rotating with the Camera cannot be measured by the LOWFSs (one component is invisible, and the other component is visible but degenerate with static height difference between the two wavefront sensors).

Both of these gradient terms were diagnosed and eliminated during commissioning. For the M1-rotator alignment, we used a specific on-sky procedure: tracking several stars past the zenith, starting from different Cassegrain rotator angles, while logging wavefront sensor readings, creates a plot of focus difference between the two LOWFSs against Cassegrain rotator angle: misaligment creates a sinusoidal dependence as above. From the amplitude and phase of this, the required M1 lateral shift can be computed, and applied manually via the lateral definer adjustment screws.

For the Camera term we used data from the science detectors: blinking pairs of images with M2 offset above/below best focus at several Cassegrain rotator angles was used to estimate the component of focus gradient co-rotating with the Camera. This tilt estimate was used to manufacture a new shim-ring between Camera and Cassegrain rotator, with a wedge angle $\approx 150 \mu \mathrm{rad}$ to correct the measured gradient.

Both of the above procedures took some time to develop and optimise; an added challenge occurred because a documentation inconsistency led the LOWFS software to initially contain a systematic focus error (dependent on the off-axis distance of the LOWFS star within the CCD). This error was of an annoying size, i.e. large enough to be significant but not large enough to be readily obvious. This created an apparent "phantom wobble" $\sim \pm 150 \mathrm{~nm}$ in focus measurements only, which confused the interpretation until it was eventually diagnosed and then easily fixed. After this fix, the procedure worked very well; focus gradient became negligible at all Cassegrain rotator angles, and has remained so. The mechanical structure between M1 and Cassegrain rotator is very rigid by design (Sect. 6), so that this one-time alignment of M1 and Camera is sufficient. A re-run of the $\mathrm{M} 1$ alignment procedure may be required after recoating of the primary mirror.

\subsection{Alignment bullseye}

It is instructive to count degrees of freedom (d.o.f.) in alignments: defining the Cassegrain axis as the datum and ignoring $z$-rotations, there are five d.o.f. each for M1, M2 and Camera, minus one for common-mode $z$-translations, hence $15-1=$ 14 d.o.f. Two of these are M1-Camera axial spacing (set by metrology) and M1-M2 spacing (closed-loop focus); the symmetry of the LOWFSs decouples these from the 12 d.o.f.'s of 
tilt and decentre of M1, M2 and Camera. In VISTA the 4 M2 tilt/decentre d.o.f's are controlled in closed loop as described above, while we did one-time corrections of M1 decentre and Camera tilt for another $2+2$ d.o.f.; this leaves the final 4 d.o.f.'s un-corrected.

A Zemax analysis revealed an interesting explanation: the analysis showed that there is a "target plane" at $z=1.02 \mathrm{~m}$ above the M1 pole, and if the combined small tilts and decenters make the M1 and Camera optical axes intersect (at a small non-zero angle) in this target plane, then the resulting 4-d.o.f. M2 compensation derived from the LOWFSs leaves the images almost identical to a fully-aligned system; coma and astigmatism are set to ideal values by the M2 compensation, and the focal gradient is also negligible for this specific combination. In practice, the axes do not have to cross exactly but it is sufficient for the M1 and Camera axes to cross this plane within $<0.2 \mathrm{~mm}$ of each other.

To ensure this condition holds at any Cassegrain rotator angle, we may then define a virtual "bullseye" of $\approx 0.1 \mathrm{~mm}$ radius centred on the rotator axis at $1.02 \mathrm{~m}$ above M1: the adjustments to M1 centering and Camera tilt described above in Sect. 8.6 effectively align the system such that both the M1 and Camera axes pass through this bullseye; it is then readily seen that the above condition will be satisfied at any rotator angle, and the active optics control of M2 will deliver near-optimal images. The 4 uncorrected d.o.f's then correspond to small rotations of M1 and Camera around the bullseye centre, which after M2 compensation do not degrade image quality at first order. (For gross combinations of tilt/decenter, second-order effects will become significant; but in practice the initial mechanical setup is good enough that tilt angles are $\lesssim 1 \mathrm{mrad}$, so it is safe to ignore effects second-order in tilt angles).

This condition essentially corresponds to the "subspace of benign misalignment" in the terminology of Schechter \& Levinson (2011), and the bullseye above provides a convenient geometrical interpretation for the VIRCAM case.

\subsection{Wavefront sensors performance}

Overall, the VIRCAM wavefront sensing system is fairly complicated, particularly with respect to software; this consumed dramatically more manpower than initially estimated in developing, testing, debugging, and modifying for robustness on the real sky.

On the positive side, the wavefront sensing has worked well since commissioning; no faults have occurred on the cold side. The system is generally reliable apart from occasional software glitches or freezes, and imposes relatively little overhead on science observations. There is no detectable interference between CCDs and science detectors. Some interference is seen between LOWFS and AG CCDs in the same unit, but only rarely when the readouts coincide by chance; this results in occasional rejected AG or LOWFS frames, but no loss of time.

\section{Enclosure and infrastructure}

VISTA is sited on its own subsidiary summit at $1.5 \mathrm{~km}$ NNE from the main Paranal summit. The mountaintop was levelled to create the platform at $2518 \mathrm{~m}$ elevation, and a new single-track road was constructed, branching off from the main VLT road.

The enclosure serves a number of purposes, including:

1. To protect the telescope from adverse weather, rain and storms.

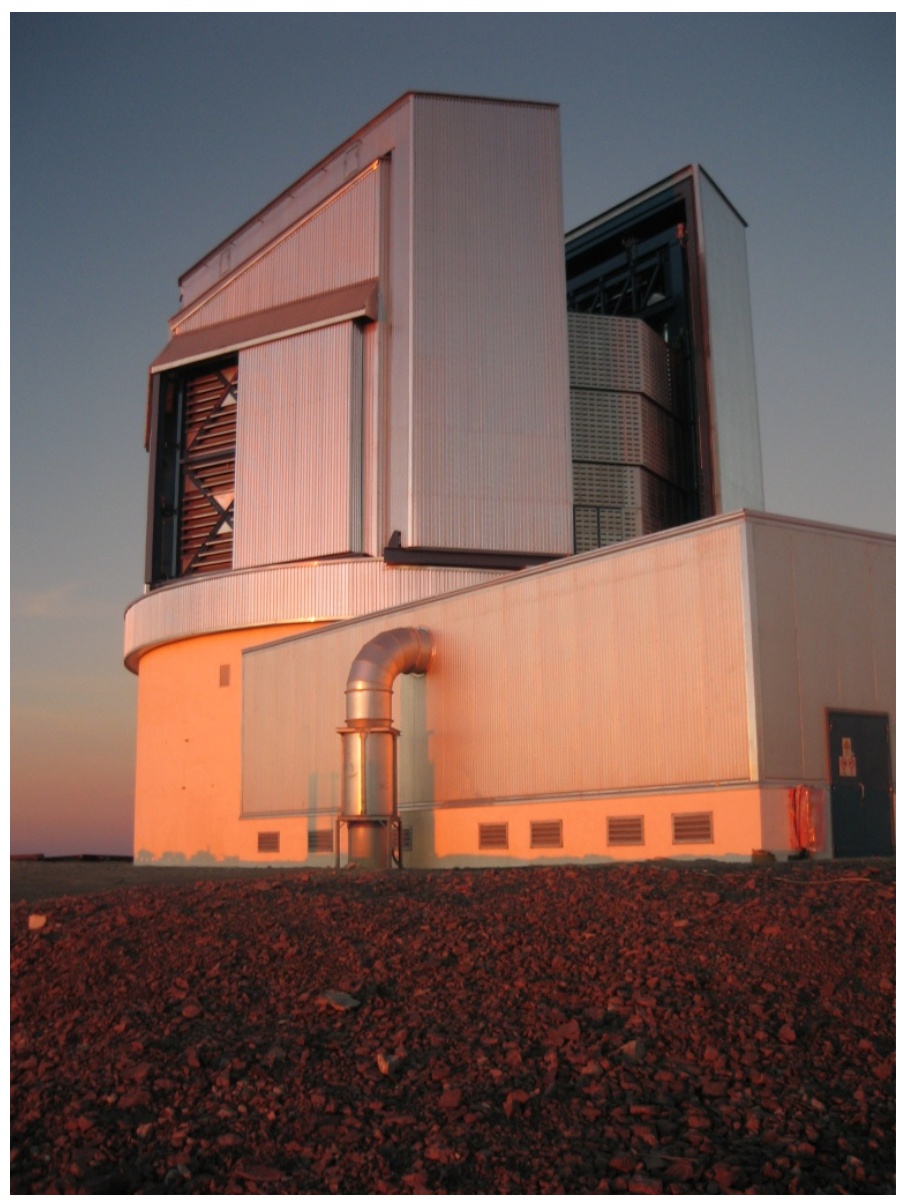

Fig. 14. VISTA enclosure seen from the southwest at sunset, with the auxiliary building in the foreground. Here the windscreen is fully raised, and an open ventilation door is seen on the left.

2. During observing, to mitigate telescope windshake in high winds, while providing good ventilation and minimal dome seeing in normal wind conditions.

3. To reduce unwanted straylight e.g. moonlight on the telescope optics.

4. To control the telescope and structures to nighttime temperatures during the day.

5. To deliver a constant-brightness light source and screen for daytime instrument calibrations.

6. To provide convenient access to the telescope during maintenance and handling operations.

7. To house the various services, including computers, electrical supply hardware, compressors, glycol pumps, etc.

The VISTA enclosure detailed design and construction was managed by EIE, Venice-Mestre. It is a relatively conventional design following modern practice for minimising local dome seeing, using a combination of active daytime cooling and good natural ventilation during observing. The dome can rotate fully independent of the telescope pointing; this is required for certain lifting operations, and is also convenient for routine daytime maintenance and testing.

The enclosure consists of three main parts (Fig. 14): the rotating octagonal "dome" housing the telescope is supported on a cylindrical concrete "base". A single-storey "auxiliary building" adjacent to the enclosure base houses the mirror coating plant, electrical supply rooms and a pump room for glycol and compressed air. 


\subsection{Enclosure base}

The enclosure base is a concrete cylinder of $18 \mathrm{~m}$ internal diameter, wall thickness $0.6 \mathrm{~m}$ and height $6.2 \mathrm{~m}$, which supports the circular dome-rotation rail on its top face. The ground floor contains six rooms around $2 / 3$ of the circumference: there are two large rooms for the local control office and the Instrument Lab respectively, and four smaller rooms for telescope electronics, Helium compressors, and a clean room if necessary to work on the detectors or electronics.

On the ground floor, a clear area of $6 \mathrm{~m}$ diameter south of the telescope pier, next to the auxiliary building, is used for M1 washing and all major lifting operations; a roll-up door connects this area to the coating plant in the auxiliary building. An entrance lobby with a $3.1 \mathrm{~m}$ wide door provides entrance for the Camera and other large items, though all planned Camera maintenance can be done in the instrument lab on the enclosure ground floor.

The enclosure base includes a concrete intermediate floor at height $4.6 \mathrm{~m}$; this area houses the four main dome air-cooling cabinets, and the slip-rings feeding power and control signals to the rotating dome. This floor also provides easy access to the telescope azimuth drives and the dome rotation mechanisms. This intermediate air volume is actively cooled both day and night, to provide a thermal buffer zone between the warm ground-level rooms below (typically $16^{\circ} \mathrm{C}$ ) and the telescope floor above.

\subsection{Dome}

The "dome" is the rotating structure housing the telescope; it is based on a regular octagon in plan, and is a steel skeleton clad with insulating panels. All parts are straight beams and planar panels for cost minimisation. The dome surfaces are clad with $80 \mathrm{~mm}$ thick insulating panels with a bare aluminium external skin, to minimise heat load on the air-cooling systems during the daytime and to minimise radiative over-cooling of the dome exterior to the cold sky at night.

The dome is built on a large octagonal steel box-beam, with a rotation bogie below each corner rolling on the $18.6 \mathrm{~m}$ diameter circular rail; a non-load-bearing circular skirt outside the octagon covers the gaps to the circular concrete base. The dome structure provides an internal clear diameter of $\approx 15 \mathrm{~m}$. Four of the bogies are motor-driven for dome rotation, and the other four are idle. Each bogie includes a single $60 \mathrm{~cm}$ main wheel, with conical surface to avoid skidding on the circular rail; four small guide-rollers maintain centration on the rail. The dome can operate normally with any three motors functional. Power and control signals are supplied by slip rings above the intermediate floor level, so the dome has unlimited rotation in either direction.

The dome includes a $5.4 \mathrm{~m}$ wide observing slit, centred at a corner of the octagon, which is closed by two inverted-L sliding doors; these slide parallel to two faces of the octagonal dome to avoid protruding "sails" when open. For earthquake resistance, the slit doors are pinned to the main structure in both the open and closed positions, by three motorised locking pins on each side of the slit. When the slit doors are closed, a seventh locking pin joins the two doors at the apex to resist gapping in extreme wind conditions. Adjoining the top of the slit are two large trusses which support the dome roof and the main enclosure crane (Fig. 1).

Six large ventilation doors (in three pairs) are placed on the other six sides of the octagon: each pair of doors provides approximately $5 \times 6 \mathrm{~m}$ open aperture, for generous natural venting of the dome in low to moderate winds. Fixed slanted louvres (see Fig. 1) are bolted to the dome skeleton inside the ventilation doors, to allow good ventilation while rejecting unwanted stray light from the Moon.

A movable windscreen is located in the lower part of the observing slit: this is a 3-panel structure with the bottom panel fixed, and 2 overlapping panels sliding upwards; the windscreen total height is adjustable from approximately $3 \mathrm{~m}$ to $7.5 \mathrm{~m}$ above the dome floor. The windscreen is porous, with fixed open slots covering about $20 \%$ of its area; this is supposed to improve ventilation when raised, and reduce vortex shedding from the upper edge.

The dome includes a deployable "moonscreen" which slides horizontally along the upper part of the slit, above the main crane. In the retracted position this is outside the telescope beam to zenith, while it can deploy forwards to fully cover a $4.5 \mathrm{~m}$ circle centred above the telescope. This moonscreen has several purposes:

1. When appropriate, to reduce illumination of the telescope optics by the Moon.

2. To provide a calibration screen for daytime measurements: the main use is for linearity calibration which requires a constant brightness source.

3. To protect the telescope optics from falling dirt or loose parts during opening/closing of the slit doors.

4. To act as a sunshade if maintenance requires opening the slit doors during daytime.

The "observing floor" (see Fig. 1) is a flat surface at $7.9 \mathrm{~m}$ above ground level, made of three sections: an inner disk is attached to the Telescope yoke; an annular fixed floor ( $3 \mathrm{~m}$ wide) is supported on steel columns from the intermediate floor, and includes the two staircases; an outer floor ring co-rotates with the Dome. Except for a fixed parking cradle for the telescope top-end, the observing floor is not required to carry major loads, so it is made of plywood over steel frame for low mass and good thermal insulation. A conductive linoleum covering provides protection against electrostatic discharge (which is significant given the very dry environment at Paranal).

One unusual feature of the Dome is the placement of the rotation rail and bogies below the observing floor level. This somewhat increases the height of the rotating Dome structure, but has several beneficial results:

1. There is no concrete to form a heat-sink above the observing floor level.

2. The slit doors and ventilation doors have their bases level with the observing floor, giving optimal ventilation and convenient maintenance.

3. The dome rotation motors are located in the intermediate floor region below the observing floor, and are simply aircooled. (The intermediate floor air volume is actively cooled both day and night, so waste heat does not accumulate there.)

4. The dome electronics cabinets on the walls also dump their heat down to the intermediate floor volume, using fans which pull in air from the dome and exhaust downwards through vents in the rotating dome floor. Thus, no glycol supply is needed to the rotating dome.

5. The placement of the rotating/fixed interface below the telescope floor means that the dome light-tightness is very good, so linearity calibration sequences can be taken during the daytime without interference from sunlight leaks.

For daytime chilling of the dome and telescope, four large vertical air-ducts are mounted on the dome walls (see Fig. 1): during 
the daytime, the dome is normally parked with the slit southfacing, where these air-ducts line up with the four glycol-fed air-cooling cabinets underneath on the intermediate floor. An inflatable seal closes the gap between the dome and the base to minimise air leakage, and three ceiling fans blow air downwards to minimise any vertical temperature gradient.

A 10-tonne capacity bridge crane is mounted from the trusses on either side of the dome slit: this crane is used for all major lifting operations, including M1, M2, and Camera lifting. The crane can traverse $\pm 2.2 \mathrm{~m}$ sideways and $\pm 6.5 \mathrm{~m}$ along the slit, including crossing above the telescope. Large items (e.g. the Camera) are carried around the telescope by slowly rotating the dome with the load suspended.

The dome also includes internal catwalks at the rear, along the sides of the slit for access to the crane, and ladders for access to the flat roof and upper slit door motors. A battery-powered scissors-lift platform is provided to reach equipment on the enclosure walls.

\subsection{Auxiliary building}

The Auxiliary building is a single-storey building, approximately $19 \mathrm{~m}$ by $14 \mathrm{~m}$, which adjoins the Enclosure base on the south side. This building houses various electrical and glycol services, and the mirror Coating Plant.

Incoming electrical power arrives as a $10 \mathrm{kV}$ supply via underground cable from the Paranal central generators at base camp: a transformer converts this into 3-phase $400 \mathrm{~V}$, then a switchboard room distributes this around the building to the various subsystems. An uninterruptable power supply (UPS) battery bank supplies the computers and electronics: this UPS does not supply the telescope or dome drives, but provides enough capacity to power all the electronics and computers for $\sim 30 \mathrm{~min}$, enabling a graceful shutdown in the event of site power failure (which is rare, but does happen once or twice per year).

A large "pump room" houses many pumps circulating chilled glycol (as 33\% glycol/water solution) to the various subsystems, and an air compressor. A main glycol pump circulates the glycol from a 1500 litre cold-tank in the pump room to a 2-head chiller unit, located on the mountainside $80 \mathrm{~m} \mathrm{SSW}$ (usually downwind) from the telescope. The chiller unit runs to maintain the cold-tank between 8 to $10^{\circ} \mathrm{C}$ below the internal air temperature setpoint, unless the dewpoint rises above that. Four smaller pumps circulate the glycol from the cold-tank to the services: two pumps feed the 4 dome air-cooling cabinets, one feeds the telescope motors, electronics and Camera, and one feeds the Helium compressors and the ground floor air-cooling unit. All these glycol pumps are actually in parallel redundant pairs, with changeover valves enabling continuous operation. This feature is important, since the 4 Helium compressors feeding the VIRCAM consume $\sim 5 \mathrm{~kW}$ electrical power each, and require continuous glycol cooling. If their glycol circulation stops, these compressors rapidly trip themselves off and the camera starts to passively warm up; this is not unsafe but interferes with observations or calibrations.

\subsection{Coating plant}

The Auxiliary building also houses the Coating Plant which is used for coating both VISTA mirrors, and (if desired) can also coat the secondary mirrors from the VLT and Auxiliary Telescopes. The coating plant was manufactured by Stainless Metalcraft (Chatteris) and comprises a stainless-steel vacuum vessel containing 3 (later 4) fixed magnetrons and a motorised mirror rotation system. The vacuum vessel comprises a spheroidal lower section, which is mounted on rails and movable horizontally into the enclosure wash area; and a conical upper section, which lifts vertically on four screw-jacks to open and close the vessel.

A vacuum of around $6 \times 10^{-6}$ mbar is achieved through a combination of a roots and rotary vane rough pump set, and two ISO 500 standard cryopumps. The plant can coat the mirrors either in Aluminium, or in protected silver for optimal infrared performance (see Sect. 9.6 for the status of silver coating).

\subsection{Handling operations}

As above, all major lifting operations use the main Enclosure roof crane, after first removing a sector of the observing floor also using the crane. For M1 re-coating, a summary of the procedure is as follows:

1. The telescope is pinned at horizon-pointing, and the Camera and then M2 are removed and stored at ground level.

2. The complete top-end structure (top ring, spiders and trusses) is detached from the Altitude ring and secured to a dedicated cradle on the observing floor.

3. A pair of 4.5 tonne counterweights are attached to the Altitude ring to restore balance of the telescope tube; then the tube is hand-cranked to zenith pointing and pinned.

4. The M1 restraint clamp and lateral supports are removed; and the M1 cover is assembled at ground level and lifted onto the M1.

5. The M1 with cover is lifted vertically from the Cell using a lifting plug passing through the Cassegrain rotator, then lowered down to the wash-stand on the ground floor.

6. The cover is lifted back up and stowed in the Cell. The M1 coating is chemically stripped, and the mirror is then rinsed.

7. The M1 is lifted clear and the wash stand is removed. Then the coating vessel lower section drives underneath the mirror, and the M1 is lowered into the vessel.

8. The coating vessel lower section carries M1 back to the coating area, and the upper section closes for the coating process.

Re-fitting is the reverse of the above.

Though there are numerous steps, the M1 does not leave the building, and the main crane does all the lifting.

\subsection{Enclosure performance}

Overall the performance of the enclosure has been good: the dome rotation is very smooth and quiet, the rotation and slit doors have never had a serious mechanical jam to date, and only very minor water leaks have occurred (now believed fixed). The design provides excellent ventilation and good protection from windshake; the air-cooling system works well and has ample power for all weather conditions, although it is rather noisy.

Moderate problems were encountered with electrical and control issues: in particular, the early system had all-independent speed servos on the four rotation motors; inconsistencies between tacho readings could lead to motors "fighting" each other then tripping out on excess current. A medium-term fix was to install larger brake resistors which made the fault rare, but eventually the control system was modified to a master-slave arrangement.

It turned out that the original auxiliary building was somewhat too small for two reasons: the original mirror wash area in 
the enclosure base (below the lifting hatch) is a busy area which could not be kept optimally clean, and also storage space was very limited so the ground floor became cluttered by various items of handling equipment. For these reasons, in 2013-14 a $9 \times 10 \mathrm{~m}$ extension was added on the far end of the auxiliary building, and the coating plant was shifted away from the telescope. This provides a dedicated clean mirror wash area inside the auxiliary building, and a new storeroom.

The initial silver coating with $\mathrm{NiCr}$ protective layer gave excellent reflectivity but was not quite durable enough, showing significant degradation after $\sim 1$ year on M1; thus, in April 2011 both mirrors were re-coated in Aluminium. As of 2014, the coating plant is being upgraded with a 4th magnetron to enable a Silicon Nitride protective overcoat based on the Gemini process, and it is hoped this should produce a long-life silver coating in the near future.

Total average power consumption is $103 \mathrm{~kW}$ (Weilenmann et al. 2010), including substantial contributions from the chiller $(35 \mathrm{~kW})$ and Helium compressors $(20 \mathrm{~kW})$.

\section{Software and electronics}

\subsection{Telescope control software and electronics}

The VISTA telescope control software mainly re-uses the ESO VLT control software, giving a "look and feel" similar to the VLT control screens, so that Paranal telescope operators may readily swap between VLTs and VISTA. This software is largely written in $\mathrm{C}++$ and runs on standard Linux PCs. Special VISTAspecific modules were written for the active optics and enclosure control; significant modifications were made to the Preset module, and minor modifications were made to the other modules where necessary (Terrett \& Stewart 2010).

The high-level control software does not control hardware directly, but sends commands to Local Control Units (LCUs) which actually control the hardware. The LCUs are small diskless computers running the VXWorks real-time operating system and programmed in $\mathrm{C}$, and there is one independent LCU per moving axis or subsystem. There are eleven LCUs in total: one for each Telescope axis, one each for M1 supports and M2 hexapod, one for VIRCAM, four for the two autoguiders and two low-order wavefront sensors, and one for the Enclosure control.

Customised electronics units are also used for the low-level control of the telescope axis servo loops; also a CANbus unit is used to control the M1 pneumatic supports, and a PMAC controller is used for the M2 Hexapod motor drives.

\subsection{Observing queue}

For the high-level observation control, the standard ESO BOB (Broker for Observation Blocks) is used; this takes Observation Blocks prepared by Phase 2 Preparation Package (P2PP) for Surveys (Bierwith et al. 2010). For tiling user-defined areas of sky, a package called the Survey Area Definition Tool (SADT) was developed: this automatically defines suitable overlapping pointings with selected guide and active optics stars, and creates an XML file fed to P2PP which populates these into observing blocks. This automation is important since each rectangular VISTA tile includes six distinct telescope pointings (pawprints) to provide uniform filling-in of detector gaps, and each pawprint in turn requires one guide star and two LOWFS stars. For redundancy, the software selects a ranked list of up to five candidate stars for each of the above, so up to $6 \times 3 \times 5=90$ candidate guide/LOWFS stars may be selected for a single tile. By default the software automatically selects the top-ranked star for each sensor, but the telescope operator can override this choice in the event of unsuitable star(s).

\section{Assembly and commissioning}

The official project kickoff was in April 2000. The Phase A design phase was closed out in September 2001, and essentially all the major contracts were in place by early 2003. During 2003 2004, the mountain peak was flattened, the new branch road was built and asphalted, and the enclosure concrete base was completed. In parallel, during 2003-2004 the final design reviews took place for the various subsystems. Generally, the progress during manufacture and assembly went as planned, though a number of subsystems arrived slightly later than planned. The enclosure was weatherproof in mid-2005, and the telescope structure was installed on site and tested throughout 2006. The complete VIRCAM arrived at Paranal in January 2007, but unfortunately at that point neither of the mirrors was completed.

A "first glimmer" was achieved in March 2007, using a small $20 \mathrm{~cm}$ Maksutov telescope mounted to the dummy instrument mass on the Cassegrain rotator, observing the sky through a pre-designed hole through the top-end barrel structure. This run proved useful to build a preliminary pointing model and tune the telescope control software, before arrival of the real mirrors.

The secondary mirror arrived on site in May 2007, followed by the primary mirror in March 2008. To save time, the primary mirror was transported by air from Moscow to Antofagasta in a specially chartered Antonov cargo aircraft. After this, an intensive period of on-sky commissioning and debugging followed. In the initial phase, a small and simple test camera (Puntino) with imager and Shack-Hartmann wavefront sensor was used, to build an initial pointing model and active optics lookup tables; then the test camera was replaced with VIRCAM in June 2008, and VIRCAM was used through the following 14 months of commissioning. During commissioning, we were pleased to find that there were no severe design flaws and the system was capable of good performance; however the commissioning process took significantly longer than planned due to a rather large number of relatively mundane technical glitches (notably wiring/connection problems, unstable power supply units and glycol supply problems), a lot of software debugging (especially on wavefront sensing) along with a few more serious snags: the latter are outlined in more detail in Emerson \& Sutherland (2010). Science verification occurred in October 2009, followed by formal handover from VISTA Consortium via STFC to ESO ownership in December 2009, as part of the UK in-kind contribution to joining ESO.

\section{Observing, data processing and archiving}

Here we give a short overview of the high-level observation software, VISTA Data Flow System (VDFS; Emerson et al. 2004) and VISTA Science Archive (VSA); this is only a brief outline and we refer to relevant papers for more details.

\subsection{Jittering and pawprints}

The VIRCAM detectors sparse-fill the focal plane, with gaps of 0.9 detector width in the $x$-direction and 0.425 detector width in the $y$-direction. For gap-free sky coverage, a set of six offset pointings (known as pawprints) gives one filled rectangular tile. One tile consists of a central rectangle $1.475 \times 1.017$ degrees covered by at least two of the six pawprints; plus two thin stripes 
each 0.092 deg wide (along the two long edges) covered by one pawprint. In practice, each pawprint is usually comprised of several or many offset jitter positions with typically $\sim 15$ arcsec offsets, for optimal removal of detector artefacts in later processing. The standard 6-point pawprint pattern (in several permutations of order) and various jitter patterns are defined as standard ESO templates, for convenience. For telescope jitter movements, the same guide and active optics stars must be re-used before and after (moving the readout windows in software), so the SADT software avoids stars falling too close to a detector edge. Moves to a new pawprint always require new guide and AO stars. Typically a jitter move takes $\approx 7 \mathrm{~s}$, and a pawprint move $\approx 10 \mathrm{~s}$. Much additional information is available in the ESO VIRCAM User Manual $^{5}$.

\subsection{Data processing}

Quick-look data processing (QC0) is carried out in real-time at Paranal (Hummel et al. 2010); this is primarily for timely assessment of data quality and detection of problems, not a final reduction. The data volume is $\sim 300 \mathrm{~GB}$ per night average; until 2012 this was sent back on USB disks by air-freight. Since 2013, Paranal has been connected by optical fibre to the main Chilean internet (delivered by the EVALSO project, Lemke et al. 2012), and the data now transfers over the internet. The standard data pipeline is run at the Cambridge Astronomical Survey Unit (CASU); full details are given in Lewis et al. (2010), and we summarise the steps here. Substantial information is available at the CASU web pages ${ }^{6}$.

1. Reset correction happens automatically in the doublecorrelated sampling, so is not specifically a processing step.

2. Dark correction is done using dark frames.

3. Linearity correction is done using polynomials, fitted to sequences of dome-flat frames.

4. Flat-field correction is done using twilight sky flats.

5. Sky background correction is done using various median operations on object-masked frames.

6. "Destriping" corrects an effect of horizontal non-repeatable stripes which are common across groups of four detectors, hence originates in the IRACE electronics.

7. Stacking: at this point the set of jittered images for a single pawprint are shifted and combined into a single stacked image with bad-pixel rejection.

After this, catalogue generation is run and the catalogues are astrometrically and photometrically calibrated using 2MASS stars.

\subsection{Archiving and access}

Processed data is archived both at VISTA Science Archive (VSA), Edinburgh, and at ESO. The VSA carries out many additional processing steps including combining processed pawprints into tiles, associating catalogue detections between different passbands, and constructing matching tables against external catalogues such as SDSS and 2MASS. The VSA user access includes a sophisticated SQL Server engine with numerous indexed variables, enabling fast processing of advanced queries.

\footnotetext{
5 The VIRCAM user manual is at http://www. eso.org/sci/facilities/paranal/instruments/ vircam.html

6 The CASU-VDFS technical information is at http://casu.ast.cam.ac.uk/surveys-projects/vista/ technical
}

Table 3. Typical VISTA system zeropoints, for broadband filters.

\begin{tabular}{lccc}
\hline \hline Filter & $\begin{array}{c}\text { Zeropoint } \\
(\text { Vega, } 1 \mathrm{ADU} / \mathrm{s})\end{array}$ & $\begin{array}{c}\text { Zeropoint } \\
\left(\text { Vega, } 1 e^{-} / \mathrm{s}\right)\end{array}$ & $\begin{array}{c}\text { AB-Vega offset } \\
(\mathrm{mag})\end{array}$ \\
\hline$Z$ & 23.95 & 25.51 & 0.52 \\
$Y$ & 23.50 & 25.06 & 0.62 \\
$J$ & 23.79 & 25.35 & 0.94 \\
$H$ & 23.89 & 25.45 & 1.38 \\
$K_{\mathrm{s}}$ & 23.06 & 24.62 & 1.84 \\
\hline
\end{tabular}

Many details of the VSA system are provided by Cross et al. (2012).

\section{System performance and public surveys}

\subsection{Performance summary}

Generally, we are pleased to report that the overall system works very well; the VISTA telescope with VIRCAM routinely delivers excellent wide-field images with sensitivity exceeding the original specifications (due notably to the high detector QE).

The system has been in routine operation since November 2009 , and the median delivered image quality is $\approx 0.9$ arcsec, with a slight trend with wavelength. The 10th-percentile values are around 0.7 arcsec, and images below 0.6 arcsec FWHM averaged across the full field are not uncommon.

The system reliability has improved as expected over time as glitches are fixed and/or workarounds are implemented to minimise impact. Technical time loss was around 10\% during the first year, but has steadily improved and is now comparable to the VLT's; this is good considering that VISTA as a singleinstrument telescope is more vulnerable to instrument faults.

Typical measured system zeropoints (for $1 \mathrm{ADU} / \mathrm{s}$ ) are given in Table 3.

\subsection{Astrometry}

The astrometric accuracy of VIRCAM images is good; mean residuals from the fifth-order distortion pattern are below 0.025 arcsec. Comparison of overlaps between different tiles indicates typical systematic (absolute) offsets $\sim 0.05$ arcsec at high galactic latitude; this is probably limited by random noise (in 2MASS) on the 20 2MASS stars used per pawprint, but is already comfortably good enough for object matching to other wavebands and followup spectroscopy. Differential astrometry within one tile is likely to be substantially better than this for sufficiently bright objects. Further calibration with e.g. the earlyrelease Gaia catalogues will reveal the ultimate astrometric accuracy achievable with VISTA.

\subsection{Photometry}

Briefly, VISTA photometry in the archive is given in the native VISTA filter system (relative to Vega); this is quite similar to the WFCAM system except for VISTA's $K_{\mathrm{s}}$ filter. The actual zeropoint for each pawprint is determined using matching 2MASS stars. Firstly, a large set of images on stable nights is used to determine mean colour equations between 2MASS and VISTA systems, and these (fixed) equations are then used to convert actual 2MASS magnitudes to predicted VISTA-system magnitudes for the 2MASS stars in a given pawprint. The median difference between predicted and instrumental VISTA magnitude (after distortion correction) is then used to set the oveall zeropoint for a 
VISTA pawprint. This procedure is good for $J, H, K_{\mathrm{s}}$, but somewhat more uncertain at $Z, Y$ where extrapolation from 2MASS is required. Additional corrections (known as "grouting") are required for a catalogue derived from a tile.

\subsection{Image artefacts}

The VIRCAM raw frames contain $\sim 1$ percent dead or hot pixels, but these are removed very well by jittering and image stacking. There is a problem with one half of Detector 16 which exhibits unstable flat-fields, particularly at $Z Y J$ bands, and is generally rejected for science data. Detector persistence is much lower than UKIRT WFCAM, but not entirely negligible for very bright stars. No cross-talk between readout channels has been observed. The most prominent artefacts are the filter ghosts around very bright stars, and also some smaller "jets" near moderately bright stars. The jets run perpendicular to detector edges, and appear to arise when a star lands a few arcsec outside a detector edge; these are suspected to be an internal reflection from structure at the detector edge. These ghosts and jets are generally easy to recognise due to the proximity of the parent star.

\subsection{Example images}

The VISTA images are very large ( $16 \mathrm{k}$ by $12 \mathrm{k}$ pixels for a filled tile image) so reproduction on A4 paper or computer screen results in drastic loss of detail. Online zoomable images are much better to preserve information content, and a selection of these are available ${ }^{7}$.

\subsection{Public surveys}

For the first five years of operations, over $75 \%$ of the VISTA observing time is being allocated to six large Public Surveys; these were selected by a dedicated ESO panel with a view both to standalone scientific merit, and also wide-ranging legacy value of the data to the general community. The remaining time is allocated to smaller PI-style programs via the standard ESO proposal process.

The six surveys comprise one Hemisphere survey; one Galactic bulge/plane survey; one Magellanic Cloud survey; and three nested extragalactic surveys with a range of area and depth. Here we just provide a short summary of each survey, with a reference to more details.

- VHS (VISTA Hemisphere Survey; McMahon et al. 2013): this covers almost the full southern Hemisphere, $\delta<0$, in at least $J$ and $K_{\mathrm{s}}$ passbands. In more detail, there are three strategies for different sub-regions. The DES survey area will be covered with $120 \mathrm{~s}$ exposures in $J, H, K_{\mathrm{s}}$ bands. The VST-ATLAS area will be covered with $60 \mathrm{~s}$ exposures in the four bands $Y, J, H, K_{\mathrm{s}}$. The remaining area (about half) will be covered with $60 \mathrm{~s}$ exposures at $J, K_{\mathrm{s}}$ only. This survey is providing the core selection for the Gaia-ESO spectroscopic survey, and will provide important near-IR photometry to complement the Gaia mission.

- VVV (VISTA Variables in Via Lactea; Saito et al. 2012). This survey covers a total of $500 \mathrm{deg}^{2}$ in the Galactic bulge and inner plane, with substantial multi-epoch coverage. The complete area was covered in all five broadbands in the 2010

\footnotetext{
7 http://www.eso.org/public/images/archive/zoomable/ ?search=vista
}

season, and subsequent seasons concentrate on multi-epoch sampling, with up to 100 epochs mainly in $K_{\mathrm{s}}$ band.

- VMC (VISTA Magellanic Cloud survey; Cioni et al. 2011). This survey covers both Magellanic Clouds and part of the Bridge, mainly in $Y, J, K_{\mathrm{s}}$ bands. The survey depth is intended to reach the main-sequence turnoff in the $K_{\mathrm{s}}$ band, except in the most crowded fields. Around 12 epochs of $K_{\mathrm{S}}$ band are obtained for variability information.

- VIKING (VISTA Kilo-degree Infrared Galaxy Survey; Edge et al. 2013). This is a medium-depth extragalactic survey of $1500 \mathrm{deg}^{2}$, approximating the $2 \mathrm{dFGRS}$ areas, with coverage matching the VST-KIDS visible survey (de Jong et al. 2013). Coverage includes all the Herschel-ATLAS survey fields except the northern field, and the GAMA redshift survey fields. An early science highlight is discovery of three quasars at $z=6.6,6.8,6.9$ respectively (Venemans et al. 2013), using NTT $i, z$ followup and and FORS spectroscopy. (These are currently the second, third and fourth highest redshift known quasars.)

- VIDEO (VISTA Deep Extragalactic Observations; Jarvis et al. 2013). This is a deep survey covering $12 \mathrm{deg}^{2}$, in three well-studied extragalactic survey fields (extended Chandra Deep Field south; XMM-LSS; and ELAIS-S1), in all five VISTA broadband filters. Exposure times per pixel are 4-8 h per passband. All fields have existing mid-IR coverage from the Spitzer SWIRE and SERVS projects, and sub-mm coverage from Herschel HERMES, and are likely to be prime targets for future SKA pathfinders and ALMA. The science goals include the galaxy population at $1<z<2.5$, high redshift clusters; low-luminosity AGNs; and identification and photometric redshifts of submm sources from the Herschel HERMES surveys.

- UltraVISTA (Ultradeep VISTA survey; McCracken et al. 2012). This is an ultra-deep survey covering a single VISTA tile, covering most of the HST-COSMOS field. Along with the UKIDSS-UDS field, this is the best-studied degree-sized region in the extragalactic sky, and has a wealth of multiwavelength data from most major ground and space observatories. The first year's observations in 2010 delivered a significant advance on previous CFHT-WIRCAM imaging sensitivity, and added the valuable $Y$ passband. Further seasons are providing substantially deeper data on half of the field (covering four disjoint stripes). A recent highlight is discovery of 4-10 good candidate $z \approx 7$ galaxies, the brightest examples known (Bowler et al. 2012).

\subsection{Longer term and $4 M O S T$}

The projected completion dates for the above VISTA public surveys are around early 2017 (subject to continued approval). For the longer term future, the ESA Euclid space mission is approved for launch around 2020. The Euclid field of view and near-IR pixel count are similar to VISTA, but given the much lower near-IR sky foreground in space, Euclid will deliver substantially fainter sensitivity relative to VISTA, by $\sim 2-3 \mathrm{mag}$. However, Euclid has several restrictions including: no $K_{\mathrm{s}}$ band; very limited planned coverage at low galactic latitudes (unless there is a substantial mission extension); lack of narrowband filter capability (quasi- $Y, J, H$ filters only); and limited monitoring capability away from the ecliptic poles (due to Sun-angle constraints). VISTA does not have these restrictions and thus offers significant complementarity.

Although spectroscopy did not form part of the original VISTA project objectives, the telescope's combination of very 
wide field and large instrument capability at Cassegrain focus turns out to be very suitable for a future large-multiplex fibre spectrograph. A second-generation VISTA instrument known as 4MOST (4-m Multi-Object Spectroscopic Telescope, de Jong et al. 2012) has been approved by ESO and is entering the preliminary design phase. The baseline specifications for 4MOST include a new 4-lens visible corrector with ADC giving 2.5 degree diameter field of view; and a multiplex of 2400 individually positioned fibres using an Echidna-style spine positioner (simultaneously 1600 fibres to two medium-resolution spectrographs, and 800 fibres to one high-resolution spectrograph). The 4MOST instrument offers very exciting science into the Euclid era, both for followup of the Gaia and eROSITA space missions, large $z \lessgtr 1$ redshift surveys, and followup of the wide-area imaging surveys (especially VHS, VIKING and VMC), with a predicted yield of $\sim 25$ million spectra in a 5 year merged science programme.

We also note that around 2.5 years of VIRCAM time will elapse between the projected completion of the public surveys above and the delivery of 4MOST, so there are likely to be opportunities for novel large projects using VIRCAM in this timeframe.

\section{Conclusions}

We have outlined the design, construction and performance of the $4.1 \mathrm{~m}$ wide-field VISTA telescope at Cerro Paranal and its 67 Mpixel infrared camera (VIRCAM), from the Phase A study in April 2000 to the start of science operations in October 2009 and early science results. We have described several of the novel design features, including the $f / 1.0$ primary, cold-baffled IR camera with dichroic baffle coating, and the 5-axis closedloop collimation system, using dual off-axis curvature wavefront sensors.

In the medium term, VISTA with VIRCAM is expected to remain the world's fastest wide-area near-infrared imaging system, and the only such system in the southern hemisphere, until the predicted launch of the ESA Euclid space mission in the early 2020's. The six ESO public surveys are making steady progress, and many interesting science results are emerging, as briefly outlined above. These surveys will be also very important resources for target selection for upcoming major facilities including ALMA, SKA pathfinders, MOONS, and JWST.

Acknowledgements. The VISTA project was made possible by funding from the UK Joint Infrastructure Fund (JIF) and PPARC (later STFC). Overall systems design and management was done by the VISTA Project Office at UKATC, Edinburgh, and the IR Camera was led by RAL Space with substantial contributions from UKATC and University of Durham. The participating institutions were Queen Mary University of London (Lead institution); Queen's University Belfast; Univ. of Birmingham; Univ. of Cambridge; Cardiff University; Univ. of Central Lancashire; Univ. of Durham; Univ. of Edinburgh; Univ. of Hertfordshire; Keele University; Univ. of Leicester; Liverpool John Moores University; Univ. of Nottingham; Univ. of Oxford; Univ. of St. Andrews; Univ. of Southampton; Univ. of Sussex; University College, London. We are grateful to early advice from ESO staff including Stefano Stanghellini, Massimo Tarenghi and Martin Cullum. Mark Casali, Sue Worswick and Martin Fisher made substantial contributions in the Phase A design studies, and the late Richard Bingham provided detailed design for the WFS/AG units. Numerous individuals served on the VISTA Science Committee (Chair: Andy Lawrence) which drafted the science requirements; project oversight was provided by the VISTA Executive Board (Chair: Mike Edmunds) and later the VISTA Project Board (Chair: Richard Wade) with Robert Laing and Pat Roche as external members. We thank the many technical experts who assisted by serving on the numerous review panels during the course of the project, and the many engineers at UKATC, RAL Space, LZOS, Vertex-RSI, Raytheon Vision Systems, NTE Barcelona and
EIE Venice who contributed major efforts in turning the project into reality. We thank many ESO staff, especially Carlos La Fuente, Andres Parraguez, Pascual Rojas, Thomas Szeifert and Valentin Ivanov for invaluable support during the commissioning phase and thereafter.

\section{References}

Abazajian, K. N., Adelman-McCarthy, J. K., Agueros, M. A., et al. 2009, ApJS, 182,543

Abdulkadyrov, M. A., Patrikeev, A. P., Belousov, S. P., et al. 2008a, Proc. SPIE, 7018, 701804

Abdulkadyrov, M. A., Patrikeev, A. P., Belousov, S. P., et al. 2008b, Proc. SPIE, 7018, 70180B

Abell, P. A., Allison, J., Anderson, S. F., et al. 2009, LSST Science Book, version 2.0 [arXiv: 0912.0201]

Bennett, R. J., \& Baine, F. 2004, Proc. SPIE, 5497, 91

Bezawada, N., Ives, D., \& Woodhouse, G. 2004, Proc. SPIE, 5499, 23

Bierwith, T., Szeifert, T., Dorigo, D., et al. 2010, Proc. SPIE, 7737, 773733

Bowler, R. A. A., Dunlop, J. S., McLure, R. J., et al. 2012, MNRAS, 426, 2772

Cappacioli, M., \& Schipani, P. 2011, ESO Messenger, 146, 2

Casali, M., Adamson, A., Alves de Oliveira, C., et al. 2007, A\&A, 467, 777

Cioni, M.-R., Clementini, G., Girardi, L., et al. 2011, A\&A, 527, A116

Clark, P., Berry, P., Bingham, R. G., et al. 2004, Proc. SPIE, 5499, 379

Craig, S. C., Atad-Ettedgui, E., Casali, M. M., et al. 2003, Proc. SPIE, 4837, 178

Cross, N. J. G., Collins, R. S., Mann, R. G., et al. 2012, A\&A, 548, A119

Cuillandre, J.-C. J., Withington, K., Hudelot, P., et al. 2012, Proc. SPIE, 8448, 84480M

Dalton, G. B., Caldwell, M., Ward, A. K., et al. 2006, Proc. SPIE, 6269, 34

Dalton, G. B., Sutherland, W. J., Emerson, J. P., et al. 2010, Proc. SPIE, 7735, 773551

de Jong, R., Bellido-Tirado, O., Chiappini, C., et al. 2012, Proc. SPIE, 8446, $84460 \mathrm{~T}$

de Jong, J. T. A., Kuijken, K., Applegate, D., et al. 2013, ESO Messenger, 154, 44

Doehring, T., Jedamzik, R., Wittmer, V., \& Thomas, A. 2004, Proc. SPIE, 5494, 340

Edeson, R. L., Shaughnessy, B. M., Whalley, M. S., Burke, K., \& Lucas, J. M. 2004, Proc. SPIE, 5497, 508

Edge, A., Sutherland, W., Kuijken, K., et al. 2013, ESO Messenger, 154, 32

Emerson, J. P., \& Sutherland, W. J. 2010, Proc. SPIE, 7733, 773306

Emerson, J. P., Irwin, M. J., Lewis, J., et al. 2004, Proc. SPIE, 5493, 401

Flaugher, B. L., Abbott, T. M. C., Angstadt, R., et al. 2012, Proc. SPIE, 8446, 844611

Geijo, E. M., Casalta, J. M., Canchado, M., et al. 2006, Proc. SPIE, 6273, 627338 Gunn, J. E., Siegmund, W. A., Mannery, E. J., et al. 2006, AJ, 131, 2332 Hummel, W., Hanuschik, R., de Bilbao, L., et al. 2010, Proc. SPIE, 7737, 773755 Jarvis, M. J., Bonfield, D. G., Bruce, V. A., et al. 2013, MNRAS, 428, 1281 Jeffers, P., Stobie, B., Hermann, S., \& McCreight, B. 2006, Proc. SPIE, 6267, 39

Keller, S. C., Schmidt, B. P., Bessell, M. S., et al. 2007, PASA, 24,

Lawrence, A., Warren, S. J., Almaini, O., et al. 2007, MNRAS, 379, 1599

Leclerc, M., Atad-Ettedgui, E., \& Gallie, A. 2004, Proc. SPIE, 5249, 449

Lemke, R., Comeron, F., Chini, R., et al. 2012, Proc. SPIE, 8451, 84511G

Lewis, J. R., Irwin, M., \& Bunclark, P. 2010, in Astronomical Data Analysis Software and Systems XIX, eds. Y. Mizumoto, K. Morita, \&, M. Ohishi, ASP Conf. Ser., 434, 91

Love, P. J., Hoffman, A. W., Ando, K. J., et al. 2004, Proc. SPIE, 5499, 379

McCracken, H. J., Milvang-Jensen, B., Dunlop, J., et al. 2012, A\&A, 544, A156 McLeod, B. A. 1996, PASP, 108, 217

McMahon, R. G., Banerji, M., \& Gonzales, E. 2013, ESO Messenger, 154, 35

Noethe, L., \& Guisard, S. 2000, A\&AS, 144, 157

Patterson, B. A., \& Wells, M. 2003, Proc. SPIE, 4842, 116

Probst, R. G., George, J. R., Daly, P. N., Don, K., \& Ellis, M. 2008, Proc. SPIE 7014, 93

Puget, P., Stadler, E., Doyon, R., et al. 2004, Proc. SPIE, 5492, 978

Saito, R. K., Hempel, M., Minnitti, D., et al. 2012, A\&A, 537, A107

Schechter, P. L., \& Levinson, R. S. 2011, PASP, 123, 812

Skrutskie, M. F., Cutri, R. M., Stiening, R., et al. 2006, AJ, 131, 1163

Stobie, B., Jeffers, P., Stewart, M., Foster, A., \& Delgadillo, J. 2010, Proc. SPIE, 7733, 77332P

Terrett, D. L., \& Stewart, M. 2010, Proc. SPIE, 7735, 77351J

Terrett, D. L., \& Sutherland, W. J. 2010, Proc. SPIE, 7740, A77403

Tokovinin, A., \& Heathcote, S. 2006, PASP, 118, 1165

Venemans, B. P., Findlay, J.R, Sutherland, W. J., et al. 2013, ApJ, 779, 24

Weilenmann, U., Ramirez, C., \& Vanderheyden, P. 2010, Proc. SPIE, 7737, $77371 \mathrm{Y}$ 